\title{
The Euler-Poincaré Equations and Double Bracket Dissipation
}

\author{
Anthony Bloch * \\ Department of Mathematics, University of Michigan \\ Ann Arbor, MI 48109 \\ P.S. Krishnaprasad ${ }^{\dagger}$ \\ Department of Electrical Engineering and \\ Institute for Systems Research \\ University of Maryland, College Park, MD 20742 \\ Jerrold E. Marsden $\ddagger$ \\ Control and Dynamical Systems, 104-44 \\ California Institute of Technology, Pasadena, CA 91125 \\ Tudor S. Ratiu ${ }^{\S}$ \\ Department of Mathematics \\ University of California, Santa Cruz, CA 95064 \\ March, 1993; this version, June 4, 1996. \\ Comm. Math. Phys., [1996] 175, 1-42.
}

\begin{abstract}
This paper studies the perturbation of a Lie-Poisson (or, equivalently an Euler-Poincaré) system by a special dissipation term that has Brockett's double bracket form. We show that a formally unstable equilibrium of the unperturbed system becomes a spectrally and hence nonlinearly unstable equilibrium after the perturbation is added. We also investigate the geometry of this dissipation mechanism and its relation to Rayleigh dissipation functions. This work complements our earlier work (Bloch, Krishnaprasad, Marsden and Ratiu [1991, 1994]) in which we studied the corresponding problem for systems with symmetry with the dissipation added to the internal variables; here it is added directly
\end{abstract}

\footnotetext{
${ }^{*}$ Research partially supported by the National Science Foundation PYI grant DMS-91-57556, and AFOSR grant F49620-93-1-0037

${ }^{\dagger}$ Research partially supported by the AFOSR University Research Initiative Program under grants AFOSR-87-0073 and AFOSR-90-0105 and by the National Science Foundation's Engineering Research Centers Program NSFD CDR 8803012

${ }^{\ddagger}$ Research partially supported by DOE contract DE-FG03-92ER-25129, a Fairchild Fellowship at Caltech, and the Fields Institute for Research in the Mathematical Sciences

${ }^{\S}$ Research partially supported by NSF Grant DMS 91-42613, DOE contract DE-FG03-92ER25129, the Fields Institute, the Erwin Schrödinger Institute, and the Miller Institute of the University of California.
} 
to the group or Lie algebra variables. The mechanisms discussed here include a number of interesting examples of physical interest such as the Landau-Lifschitz equations for ferromagnetism, certain models for dissipative rigid body dynamics and geophysical fluids, and certain relative equilibria in plasma physics and stellar dynamics.

\section{Contents}

1 Introduction 2

2 Motivating Examples 5

3 Dissipative Systems $\quad 8$

4 Equivariant Dissipation $\quad 13$

5 The Euler-Poincaré equations $\quad 14$

6 Dissipation for Euler-Poincaré and Lie-Poisson Equations 18

7 The Lie-Poisson Instability Theorem 23

8 Lie-Poisson Examples $\quad \mathbf{2 6}$

8.1 The Rigid Body and the Landau-Lifschitz equations . . . . . . . . . 26

8.2 Ideal Fluids . . . . . . . . . . . . . . . . . . . . 26

8.3 The Vlasov-Poisson Equations . . . . . . . . . . . . . . . . . . . 28

8.4 The Heavy Top . . . . . . . . . . . . . . . . . . . . . . . . 30

9 Instability for Systems with both Internal and Double Bracket Dis$\begin{array}{ll}\text { sipation } & 31\end{array}$

10 The Rigid body with rotors $\quad 34$

$\begin{array}{ll}11 \text { Conclusions and Comments } & 37\end{array}$

12 Appendix. The Euler-Poincaré Equations for General Lie Groups 38

\section{Introduction}

The purpose of this paper is to study the phenomenon of dissipation induced instabilities for Euler-Poincaré systems on Lie algebras or equivalently, for Lie-Poisson systems on the duals of Lie algebras. Lie-Poisson systems on the duals of Lie algebras $\mathfrak{g}^{*}$ are obtained by reduction from invariant Hamiltonian systems on cotangent bundles $T^{*} G$ of the corresponding Lie group and, as we shall recall later, EulerPoincaré systems on Lie algebras $\mathfrak{g}$ are the reductions of invariant Euler-Lagrange equations on the associated tangent bundels $T G$. In addition to this, we study the more general problem of adding dissipation to reduced systems with symmetry that 
come from an invarian system with a configuration manifold $Q$ on which a Lie group $G$ acts.

In our previous work (Bloch, Krishnaprasad, Marsden and Ratiu [1994], denoted hereafter by $[\mathrm{BKMR}])$, we showed that if a mechanical system with symmetry has an indefinite second variation of the augmented Hamiltonian at a relative equilibrium, as determined by the energy-momentum method (Simo, Posbergh and Marsden [1990,1991], Simo, Lewis and Marsden [1991], Lewis [1992], and Wang and Krishnaprasad [1992]), then the system becomes spectrally unstable with the addition of a small amount of internal dissipation. This energy momentum method is an outgrowth of the energy-Casimir, or Arnold method that has its roots in original work going back to at least Routh [1877]; see Holm, Marsden, Ratiu and Weinstein [1985] and references therein. The dissipation that was considered in our earlier paper was of the standard Rayleigh dissipation type, and this dissipation was added to the internal variables of the system. The methods that were used to prove this were essentially those of linear analysis. In that paper, we did not consider dissipation terms in the group (or rotational) variables; that is the subject of the present work.

For systems on Lie algebras, or equivalently, for invariant systems on Lie groups, we show that one cannot have linear dissipative terms of Rayleigh dissipation type in the equations in the naive sense. However, when restricted to coadjoint orbits, we show that these dissipation terms are obtainable from a gradient structure that is similar in spirit to the way one gets dissipative terms from the gradient of a Rayleigh dissipation function. Thus, these functions on the coadjoint orbits play the role of the Rayleigh dissipation function. In this context, we prove that one gets dissipation induced instabilities, as one does in the case of internal dissipation. This means that the addition of dissipation to a state that is a saddle point of the augmented Hamiltonian forces at least one pair of eigenvalues into the right half plane, which one refers to as spectral instability and which, of course implies nonlinear instability.

The dissipation that we construct has the essential feature that energy is dissipated but angular momentum is not. In the context of Euler-Poincaré or Lie-Poisson systems, this means that the coadjoint orbits remain invariant, but on them the energy is decreasing along orbits. Many physical systems act this way, such as dampers in satellites and dissipation due to radiation in stars.

One of the interesting features of the present work is the geometry behind the construction of the nonlinear dissipative terms which involves the double bracket equation of Brockett (see Brockett $[1988,1993]$ ). In fact, this form is well adapted to the study of dissipation on Lie groups since it was originally constructed as a gradient system and it is well known in other contexts that this formalism plays an important role in the study of integrable systems (see, for example, Bloch, Flaschka and Ratiu [1990] and Bloch, Brockett and Ratiu [1992]).

We will also show that this type of dissipation can be described in terms of a symmetric Poisson bracket. Symmetric brackets for dissipative systems have been considered by Kaufman [1984, 1985], Grmela [1984,1993a,b], Morrison [1986], and Turski and Kaufman [1987]. It is not clear how the brackets of the present paper are related to those. Our brackets are more directly motivated by those in Vallis, 
Carnevale, and Young [1989], Shepherd [1992] and references therein.

We present a class of symmetric brackets that are systematically constructed in a general Lie algebraic context. We hope that our construction might shed light on possible general properties that these brackets might have. The general equations of motion for systems with dissipation that we consider have the following form:

$$
\dot{F}=\{F, H\}-\{\{F, H\}\}
$$

where $H$ is the total energy of the system, $\{F, H\}$ is a skew symmetric bracket which is a Poisson bracket in the usual sense and where $\{\{F, H\}\}$ is a symmetric bracket. In many cases however, especially those involving thermodynamics, one replaces $H$ in the second bracket by $S$, the entropy, as in the preceding references. It remains for the future to link that work more closely with the present context and to see in what sense, if any, the combined bracket satisfies a graded form of Jacobi's identity.

The type of dissipation described here arises in several important physical contexts. First of all, some physically arising dissipative mechanisms are of this type. For example, as we shall point out below, the Landau-Lifschitz (or Gilbert) dissipative mechanism in ferromagnetics is exactly of the type we describe and this dissipative mechanism is regarded as a good model of the physical dissipation (see O'Dell [1981] for example). In geophysical situations, one would like a dissipative mechanism that separates the different time scales of decay of the energy and the enstrophy. That is, one would like a dissipative mechanism for which the energy decays but the enstrophy remains preserved. This is exactly the sort of dissipative mechanism described here and that was described in Vallis, Carnevale, and Young [1989], Shepherd [1992] and references therein. Also, in plasma physics and stellar dynamics, one would like to have a dissipative mechanism that preserves the underlying conservation of particle number, yet has energy decay. Again, the general mechanism here satisfies these properties (see Kandrup [1991] and Kandrup and Morrison [1992]). We will discuss all of these examples in the body of the paper.

This theory is also of interest in control systems. An interesting example we consider is the rigid body with internal rotors. In this example there are feedback laws that stabilize an otherwise unstable motion, such as steady rotation about the middle axis of the rigid structure, as has been shown by Bloch, Krishnaprasad, Marsden and Sanchez de Alvarez [1992] and references therein. However, the dissipation here allows one to modify this feedback so that the stability becomes asymptotic stability. In the case of compact matrix groups for concreteness, we know that for the forcing to be tangent to the (co)adjoint orbits at a point $X$, they should be of the form $[X, U]$ and for them to be of double bracket form, $U$ should itself be a bracket, say $U=[X, N]$. This $U$ then determines the required feedback law. Some related work in this general direction is given by Kammer and Gray [1993], Posbergh and Zhao [1993] and Posbergh [1994]. We think that the dissipative mechanisms here should be useful for a variety of similar control problems where it is clear from the start that controls are capable of dissipating energy, but not the total angular momentum. 


\section{Some terminology}

Since there is often confusion in the literature about terms like stability, we shall explain how the terms are used in this paper.

Stability or nonlinear stability refers to stability in the standard Liapunov sense for a given dynamical system. If it is given in the context of a fixed point, this means that the fixed point is Liapunov stable in the standard sense (initial conditions starting in a small neighborhood stay in a given neighborhood for all forward time). If it is applied to another invariant set, such as a trajectory, it means Liapunov stability for that set.

Instability means stability fails.

Spectral stability of a fixed point means that the spectrum of the linearized equations at that point lies in the strict left half plane

Spectral instability of a fixed point means that there is some eigenvalue of the linearized equations at the fixed point that lies in the strict right half plane.

Linearized stability (or instability) means that the point zero for the equations linearized at the fixed point is Liapunov stable (or unstable).

Of course it is standard that spectral stability (or instability) imples linear and nonlinear stability (or instability). However, as is well known, linear stability need not imply nonlinear stability.

\section{Summary of the Main Results}

- The construction of a general class of dissipative mechanical systems with symmetry that dissipate energy but that preserve the momentum map.

- The dissipation constructed is shown to be of double bracket form. A number of geometric properties of this dissipation are established, such as the existence of Rayleigh dissipation functions on each reduced space (such as coadjoint orbits).

- It is shown how our construction fits into a general framework for Lagrangian systems with forces described by the Lagrange-d'Alembert principle.

- The derivation of the reduced variational principle for the Euler-Poincaré equations is given (in the appendix) for general Lie groups. This is important, both for the generality achieved and since most infinite dimensional groups, such as those arising in fluid mechanics, are not matrix groups.

- General instability theorems are given; they say, roughly speaking, that when systems have relative equilibria that are saddle points of the augmented Hamiltonian, then they are spectrally (and hence linearly and nonlinearly) destabilized by the addition of a small amount of dissipation of double bracket form. 
This was proved in $[\mathrm{BKMR}]$ for internal dissipation and is extended to double bracket dissipation (which, in many examples is external dissipation) and combinations of the two in this paper.

- Several examples are studied. One of the simplest is the Landau-Lifschitz equations where double bracket dissipation is well documented from the physical point of view. We also show that our approach applies to fluid and plasma systems and work out the dissipative terms in these cases. While this type of dissipation is less well understood from the point of view of physics, it has been discussed in the literature and we expect that it is of interest in some fluid and plasma situations.

\section{Motivating Examples}

To get a concrete idea of the type of dissipative mechanism we have in mind, we now give a simple example of it for perhaps the most basic of Euler-Poincaré, or LiePoisson systems, namely the rigid body. Here, the Lie algebra in question is that of the rotation group; that is, Euclidean three space $\mathbb{R}^{3}$ interpreted as the space of body angular velocities $\Omega$ equipped with the cross product as the Lie bracket. On this space, we put the standard kinetic energy Lagrangian $L(\Omega)=\frac{1}{2}(I \Omega) \cdot \Omega$ (where $I$ is the moment of inertia tensor) so that the general Euler-Poincaré equations (discussed below in $\S 4$ ) become the standard rigid body equations for a freely spinning rigid body:

$$
I \dot{\Omega}=(I \Omega) \times \Omega,
$$

or, in terms of the body angular momentum $M=I \Omega$,

$$
\dot{M}=M \times \Omega \text {. }
$$

In this case, the energy equals the Lagrangian; $E(\Omega)=L(\Omega)$ and energy is conserved by the solutions of (2.1). Now we modify the equations by adding a term cubic in the angular velocity:

$$
\dot{M}=M \times \Omega+\alpha M \times(M \times \Omega),
$$

where $\alpha$ is a positive constant.

A related example is the 1935 Landau-Lifschitz equations for the magnetization vector $M$ in a given magnetic field $B$ (see, for example, O'Dell [1981], page 41 and Helman, Braun, Broz and Baltensperger [1991]):

$$
\dot{M}=\gamma M \times B+\frac{\lambda}{\|M\|^{2}}(M \times(M \times B)),
$$

where $\gamma$ is the magneto-mechanical ratio (so that $\gamma\|B\|$ is the Larmour frequency) and $\lambda$ is the damping coefficient due to domain walls. Similar remarks will apply to the PDE form of the equations. Some interesting computational aspects of the Landau-Lifschitz-Gilbert equations are given in Giles, Patterson, Bagneres, Kotiuga, Humphrey and Mansuripur [1991]. 
Note that in (2.3) $B$ is regarded as given whereas in (2.1) $M$ and $\Omega$ are related by $M=I \Omega$. In each case, it is well known that the equations without damping can be written in either Euler-Poincaré form or in Lie-Poisson (Hamiltonian) form. The equations are Hamiltonian with the rigid body Poisson bracket:

$$
\{F, K\}_{\mathrm{rb}}(M)=-M \cdot[\nabla F(M) \times \nabla K(M)]
$$

with Hamiltonians given respectively by $H(M)=(M \cdot \Omega) / 2$ and $H(M)=\gamma M \cdot B$.

One checks in each case that the addition of the dissipative term has a number of interesting properties. First of all, this dissipation is derivable from an $S O(3)$ invariant force field, but it is not induced by any Rayleigh dissipation function in the literal sense (we shall precisely formulate a general result along these lines later). However, it is induced by a dissipation function in the following restricted sense: It is a gradient when restricted to each momentum sphere (coadjoint orbit) where each sphere carries a special metric (later to be called the normal metric). Namely, the extra dissipative term in (2.2) equals the negative gradient of the Hamiltonian with respect to the following metric on the sphere. Take a vector $\mathbf{v}$ in $\mathbb{R}^{3}$ and orthogonally decompose it in the standard metric on $\mathbb{R}^{3}$ into components tangent to the sphere $\|M\|^{2}=c^{2}$ and vectors orthogonal to this sphere:

$$
\mathbf{v}=\frac{M \cdot \mathbf{v}}{c^{2}} M-\frac{1}{c^{2}}[M \times(M \times \mathbf{v})] .
$$

The metric on the sphere is chosen to be $\|M\|^{-2} \alpha$ times the standard inner product of the components tangent to the sphere in the case of the rigid body model and just $\lambda$ times the standard metric in the case of the Landau-Lifschitz equations.

Secondly, the dissipation added to the equations has the obvious form of a repeated Lie bracket, i.e., a double bracket, and it has the properties that the conservation law

$$
\frac{d}{d t}\|M\|^{2}=0
$$

is preserved by the dissipation (since the extra force is orthogonal to $M$ ) and the energy is strictly monotone except at relative equilibria. In fact, we have

$$
\frac{d}{d t} E=-\alpha\|M \times \Omega\|^{2}
$$

for the rigid body and

$$
\frac{d}{d t} E=-\frac{\lambda}{\|M\|^{2}}\|M \times B\|^{2},
$$

in the case of the Landau-Lifschitz equations, so that trajectories on the angular momentum sphere converge to the minimum (for $\alpha$ and $\lambda$ positive) of the energy restricted to the sphere, apart from the set of measure zero consisting of orbits that are relative equilibria or are the stable manifolds of the perturbed saddle point.

Another interesting feature of these dissipation terms is that they can be derived from a symmetric bracket in much the same way that the Hamiltonian equations 
can be derived from a skew symmetric Poisson bracket. For the case of the rigid body, this bracket is

$$
\{\{F, K\}\}=\alpha(M \times \nabla F) \cdot(M \times \nabla K) .
$$

As we have already indicated, the same formalism can be applied to other systems as well. In fact, later in the paper we develop an abstract construction for dissipative terms with the same general properties as the above examples. When this method is applied to fluids one gets a dissipative mechanism related to that of Vallis, Carnevale, and Young [1989] and Shepherd [1992] as follows. One modifies the Euler equations for a perfect fluid, namely

$$
\frac{\partial v}{\partial t}+v \cdot \nabla v=-\nabla p
$$

where $v$ is the velocity field, assumed divergence free and parallel to the boundary of the fluid container, and where $p$ is the pressure. With dissipation, the equations become:

$$
\frac{\partial v}{\partial t}+v \cdot \nabla v=-\nabla p+\alpha \mathbf{P}\left[\left(£_{u(v)} v^{b}\right)^{\sharp}\right],
$$

where $\alpha$ is a positive constant, $\mathbf{P}$ is the Hodge projection onto the divergence free part, $£$ denotes the Lie derivative, and where

$$
u(v)=\mathbf{P}\left[\left(£_{v} v^{b}\right)^{\sharp}\right] .
$$

The flat and sharp symbols denote the index lowering and raising operators induced by the metric; that is, the operators that convert vectors to one forms and vice versa. Written in terms of the vorticity, these equations become

$$
\frac{d}{d t} \omega+£_{v} \omega=\alpha £_{u(v)} \omega .
$$

This dissipative term preserves the coadjoint orbits, that is, the isovortical surfaces (in either two or three dimensions, or in fact, on any Riemannian manifold), and with it, the time derivative of the energy is strictly negative (except at equilibria, where it is zero). As we shall see, there is a similar dissipative term in the case of the Vlasov-Poisson equation for plasma physics.

\section{Dissipative Systems}

For later use, it will be useful to recall some of the basic and essentially well known facts about dissipative mechanical systems. Let $Q$ be a manifold, possibly infinite dimensional, $L: T Q \rightarrow \mathbb{R}$ be a smooth function, and let $\tau: T Q \rightarrow Q$ be the tangent bundle projection. Let $\mathbb{F} L: T Q \rightarrow T^{*} Q$ be the fiber derivative of $L$; recall that it is defined by

$$
\langle\mathbb{F} L(v), w\rangle=\left.\frac{d}{d \epsilon}\right|_{\epsilon=0} L(v+\epsilon w),
$$


where $\langle$,$\rangle denotes the pairing between the tangent and cotangent spaces. We also$ recall that the vertical lift of a vector $w \in T_{q} Q$ along $v \in T_{q} Q$ is defined by

$$
\operatorname{vert}_{v}(w)=\left.\frac{d}{d \epsilon}\right|_{\epsilon=0}(v+\epsilon w) \in T_{v} T Q .
$$

The action and energy of $L$ are defined by

$$
A(v)=\langle\mathbb{F} L(v), v\rangle
$$

and

$$
E(v)=A(v)-L(v) .
$$

Let $\Omega_{L}=(\mathbb{F} L)^{*} \Omega$ denote the pull back of the canonical symplectic form on $T^{*} Q$ by the fiber derivative of $L$; we also let $\Theta$ denote the canonical one form on $T^{*} Q$ with the sign conventions

$$
\Theta(\alpha) \cdot w=\langle\alpha, T \pi(w)\rangle,
$$

where $\alpha \in T^{*} Q, w \in T_{\alpha}\left(T^{*} Q\right)$ and $\pi: T^{*} Q \rightarrow Q$ is the canonical cotangent bundle projection. In our conventions, $\Omega=-\mathbf{d} \Theta$ so that if $\Theta_{L}$ denotes the pull back of $\Theta$ by the fiber derivative, then $\Omega_{L}=-\mathbf{d} \Theta_{L}$.

A vector field $Z$ on $T Q$ is called a Lagrangian vector field for $L$ if

$$
\mathbf{i}_{Z} \Omega_{L}=\mathbf{d} E,
$$

where $\mathbf{i}_{Z}$ denotes the operation of interior multiplication (or contraction) by the vector field $Z$. In this generality, $Z$ need not exist, nor be unique. However, we will assume throughout that $Z$ is a second order equation; that is, $T \tau \circ Z$ is the identity on $T Q$. A second order equation is a Lagrangian vector field if and only if the EulerLagrange equations hold in local charts. We note that, by skew symmetry of $\Omega_{L}$, energy is always conserved; that is, $E$ is constant along an integral curve of $Z$. We also recall that the Lagrangian is called regular if $\Omega_{L}$ is a (weak) symplectic form; that is, if it is nondegenerate. This is equivalent to the second fiber derivative of the Lagrangian being, in local charts, also weakly nondegenerate. In the regular case, if the Lagrangian vector field exists, it is unique, and is given by the Hamiltonian vector field with energy $E$ relative to the symplectic form $\Omega_{L}$. If, in addition, the fiber derivative is a global diffeomorphism, then $Z$ is the pull back by the fiber derivative of the Hamiltonian vector field on the cotangent bundle with Hamiltonian $H=E \circ(\mathbb{F} L)^{-1}$. It is well known how one can pass back and forth between the Hamiltonian and Lagrangian pictures in this hyperregular case (see, for example, Abraham and Marsden [1978]).

We now turn to the definition of a dissipative system. Consider a general Lagrangian vector field $Z$ for a (not necessarily regular) Lagrangian on $T Q$. A vector field $Y$ on $T Q$ is called weakly dissipative provided that it is vertical (i.e., $T \tau \circ Y=0)$ and if, at each point of $T Q$,

$$
\langle\mathbf{d} E, Y\rangle \leq 0 \text {. }
$$


If the inequality is pointwise strict at each nonzero $v \in T Q$, then we say that the vector field $Y$ is dissipative. A dissipative Lagrangian system on $T Q$ is a vector field of the form $X=Z+Y$, where $Z$ is a (second order) Lagrangian vector field and $Y$ is a dissipative vector field. We use the word "weak" as above. It is clear by construction that the time derivative of the energy along integral curves of $X$ is nonpositive for weakly dissipative systems, and is strictly negative at nonzero points for dissipative systems. Define the one form $\Delta^{Y}$ on $T Q$ by

$$
\Delta^{Y}=-\mathbf{i}_{Y} \Omega_{L} .
$$

Proposition 3.1 If $Y$ is vertical, then $\Delta^{Y}$ is a horizontal one-form, i.e., $\Delta^{Y}(U)=$ 0 for any vertical vector field $U$ on $T Q$. Conversely, given a horizontal one form $\Delta$ on $T Q$, and assuming that $L$ is regular, the vector field $Y$ on $T Q$ defined by $\Delta=-\mathbf{i}_{Y} \Omega_{L}$, is vertical.

Proof This follows from a straightforward calculation in local coordinates. We use the fact that a vector field $Y(u, e)=\left(Y_{1}(u, e), Y_{2}(u, e)\right)$ is vertical if and only if the first component $Y_{1}$ is zero, and the local formula for $\Omega_{L}$ (see, for example, Abraham and Marsden [1978], Section 3.5):

$$
\begin{aligned}
& \left.\Omega_{L}(u, e)\left(Y_{1}, Y_{2}\right),\left(U_{1}, U_{2}\right)\right) \\
& =\mathbf{D}_{1}\left(\mathbf{D}_{2} L(u, e) \cdot Y_{1}\right) \cdot U_{1}-\mathbf{D}_{1}\left(\mathbf{D}_{2} L(u, e) \cdot U_{1}\right) \cdot Y_{1} \\
& \quad+\mathbf{D}_{2} \mathbf{D}_{2} L(u, e) \cdot Y_{1} \cdot U_{2}-\mathbf{D}_{2} \mathbf{D}_{2} L(u, e) \cdot U_{1} \cdot Y_{2} .
\end{aligned}
$$

This shows that $\left(\mathbf{i}_{Y} \Omega_{L}\right)(U)=0$ for all vertical $U$ is equivalent to

$$
\mathbf{D}_{2} \mathbf{D}_{2} L(u, e)\left(U_{2}, Y_{1}\right)=0 .
$$

If $Y$ is vertical, this is clearly true. Conversely if $L$ is regular, and the last displayed equation is true, then $Y$ must be vertical.

Proposition 3.2 Any fiber preserving map $F: T Q \rightarrow T^{*} Q$ over the identity induces a horizontal one-form $\tilde{F}$ on $T Q$ by

$$
\tilde{F}(v) \cdot V_{v}=\left\langle F(v), T_{v} \tau\left(V_{v}\right)\right\rangle,
$$

where $v \in T Q$, and $V_{v} \in T_{v}(T Q)$. Conversely, formula (3.9) defines, for any horizontal one-form $\tilde{F}$, a fiber preserving map $F$ over the identity. Any such $F$ is called a force field and thus in the regular case, any vertical vector field $Y$ is induced by a force field.

Proof Given $F$, formula (3.9) clearly defines a smooth one-form $\tilde{F}$ on $T Q$. If $V_{v}$ is vertical, then the right hand side of formula (3.9) vanishes, and so $\tilde{F}$ is a horizontal one-form. Conversely, given a horizontal one-form $\tilde{F}$ on $T Q$, and given $v, w \in T_{q} Q$, let $V_{v} \in T_{v}(T Q)$ be such that $T_{v} \tau\left(V_{v}\right)=w$. Then define $F$ by formula (3.9); i.e., $\langle F(v), w\rangle=\tilde{F}(v) \cdot V_{v}$. Since $\tilde{F}$ is horizontal, we see that $F$ is well-defined, and its expression in charts shows that it is smooth. 
Corollary 3.3 A vertical vector field $Y$ on $T Q$ is dissipative if and only if the force field $F^{Y}$ that it induces satisfies $\left\langle F^{Y}(v), v\right\rangle<0$ for all nonzero $v \in T Q(\leq 0$ for the weakly dissipative case).

Proof Let $Y$ be a vertical vector field. By Proposition 3.1, $Y$ induces a horizontal one-form $\Delta^{Y}=-\mathbf{i}_{Y} \Omega_{L}$ on $T Q$ and, by Proposition $\mathbf{3 . 2}, \Delta^{Y}$ in turn induces a force field $F^{Y}$ given by

$$
\left\langle F^{Y}(v), w\right\rangle=\Delta^{Y}(v) \cdot V_{v}=-\Omega_{L}(v)\left(Y(v), V_{v}\right),
$$

where $T \tau\left(V_{v}\right)=w$ and $V_{v} \in T_{v}(T Q)$. If $Z$ denotes the Lagrangian system defined by $L$, we get

$$
\begin{aligned}
(\mathbf{d} E \cdot Y)(v) & =\left(\mathbf{i}_{Z} \Omega_{L}\right)(Y)(v)=\Omega_{L}(Z, Y)(v) \\
& =-\Omega_{L}(v)(Y(v), Z(v)) \\
& =\left\langle F^{Y}(v), T_{v} \tau(Z(v))\right\rangle \\
& =\left\langle F^{Y}(v), v\right\rangle
\end{aligned}
$$

since $Z$ is a second order equation. We conclude that $\mathbf{d} E \cdot Y<0$ if and only if $\left\langle F^{Y}(v), v\right\rangle<0$ for all $v \in T Q$, which gives the result.

Definition 3.4 Given a dissipative vector field $Y$ on $T Q$, let $F^{Y}: T Q \rightarrow T^{*} Q$ be the induced force field. If there is a function $R: T Q \rightarrow \mathbb{R}$ such that $F^{Y}$ is the fiber derivative of $-R$, then $R$ is called a Rayleigh dissipation function.

In this case, dissipativity of $Y$ reads $\mathbf{D}_{2} R(q, v) \cdot v>0$. Thus, if $R$ is linear in the fiber variable, the Rayleigh dissipation function takes on the classical form $\langle\mathcal{R}(q) v, v\rangle$, where $\mathcal{R}(q): T Q \rightarrow T^{*} Q$ is a bundle map over the identity that defines a symmetric positive definite form on each fiber of $T Q$.

Treating $\Delta^{Y}$ as the exterior force one-form acting on a mechanical system with a Lagrangian $L$, we now will write the governing equations of motion. The basic principle is of course the Lagrange-d'Alembert principle. First, we recall the definition from Vershik and Faddeev [1981] and Wang and Krishnaprasad [1992].

Definition 3.5 The Lagrangian force associated with a given Lagrangian $L$ and a given second order vector field $X$ is the horizontal one form on $T Q$ defined by

$$
\Phi_{L}(X)=\mathbf{i}_{X} \Omega_{L}-\mathbf{d} E .
$$

Given a horizontal one form $\omega$ (referred to as the exterior force one form), the local Lagrange d'Alembert principle states that

$$
\Phi_{L}(X)+\omega=0 .
$$

It is easy to check that $\Phi_{L}(X)$ is indeed horizontal if $X$ is second order. Conversely, if $L$ is regular and if $\Phi_{L}(X)$ is horizontal, then $X$ is second order. One can also formulate an equivalent principle in variational form. 
Definition 3.6 Given a Lagrangian $L$ and a force field $F$ (as defined in Proposition 3.2), the integral Lagrange d'Alembert principle for a curve $q(t)$ in $Q$ is

$$
\delta \int_{a}^{b} L(q(t), \dot{q}(t)) d t+\int_{a}^{b} F(q(t), \dot{q}(t)) \cdot \delta q d t=0,
$$

where the variation is given by the usual expression

$$
\begin{aligned}
\delta \int_{a}^{b} L(q(t), \dot{q}(t)) d t & =\int_{a}^{b}\left(\frac{\partial L}{\partial q^{i}} \delta q^{i}+\frac{\partial L}{\partial \dot{q}^{i}} \frac{d}{d t} \delta q^{i}\right) d t \\
& =\int_{a}^{b}\left(\frac{\partial L}{\partial q^{i}}-\frac{d}{d t} \frac{\partial L}{\partial \dot{q}^{i}}\right) \delta q^{i} d t .
\end{aligned}
$$

for a given variation $\delta q$ (vanishing at the endpoints).

In this expression, we have employed coordinate notation so that the coordinates of $q$ are denoted $q^{1}, q^{2}, \ldots, q^{n}$ or $q^{i}, i=1, \ldots, n$, and there is an implied summation over repeated indices. However, it should be noted that this coordinate notation is intended for the finite dimensional case, and one should note that the developments here apply to infinite dimensional problems as well, such as fluids and plasmas.

The two forms of the Lagrange d'Alembert principle are equivalent. This follows from the fact that both give the Euler-Lagrange equations with forcing in local coordinates (provided that $Z$ is second order). We shall see this in the following development.

Proposition 3.7 Let the exterior force one-form $\omega$ be associated to a vertical vector field $Y$, i.e., let $\omega=\Delta^{Y}=-\mathbf{i}_{Y} \Omega_{L}$. Then $X=Z+Y$ satisfies the local Lagranged'Alembert principle. Conversely, if, in addition, $L$ is regular, the only second order vector field $X$ satisfying the local Lagrange-d'Alembert principle is $X=Z+Y$.

Proof For the first part, the equality $\Phi_{L}(X)+\omega=0$ is a simple verification. For the converse, we already know that $X$ is a solution, and uniqueness is guaranteed by regularity.

To develop the differential equations associated to $X=Z+Y$, we take $\omega=$ $-\mathbf{i}_{Y} \Omega_{L}$ and note that, in a coordinate chart, $Y(q, v)=\left(0, Y_{2}(q, v)\right)$ since $Y$ is vertical, i.e., $Y_{1}=0$. ¿From the local formula for $\Omega_{L}$, we get

$$
\omega(q, v) \cdot(u, w)=\mathbf{D}_{2} \mathbf{D}_{2} L(q, v) \cdot Y_{2}(q, v) \cdot u .
$$

Letting $X(q, v)=\left(v, X_{2}(q, v)\right)$, one finds that

$\Phi_{L}(X)(q, v) \cdot(u, w)=\left(-\mathbf{D}_{1}\left(\mathbf{D}_{2} L(q, v) \cdot\right) \cdot v-\mathbf{D}_{2} \mathbf{D}_{2}(q, v) \cdot X_{2}(q, v)+\mathbf{D}_{1} L(q, v)\right) \cdot u$.

Thus, the local Lagrange-d'Alembert principle becomes

$-\mathbf{D}_{1}\left(\mathbf{D}_{2} L(q, v) \cdot\right) \cdot v-\mathbf{D}_{2} \mathbf{D}_{2} L(q, v) \cdot X_{2}(q, v)+\mathbf{D}_{1} L(q, v)+\mathbf{D}_{2} \mathbf{D}_{2} L(q, v) \cdot Y_{2}(q, v)=0$ 
Setting $v=d q / d t$ and $X_{2}(q, v)=d v / d t$, the preceding relation and the chain rule gives

$$
\frac{d}{d t}\left(\mathbf{D}_{2} L(q, v)\right)-\mathbf{D}_{1} L(q, v)=\mathbf{D}_{2} \mathbf{D}_{2} L(q, v) \cdot Y_{2}(q, v)
$$

which, in finite dimensions, reads,

$$
\frac{d}{d t}\left(\frac{\partial L}{\partial \dot{q}^{i}}\right)-\frac{\partial L}{\partial q^{i}}=\frac{\partial^{2} L}{\partial \dot{q}^{i} \partial \dot{q}^{j}} Y^{j}\left(q^{k}, \dot{q}^{k}\right) .
$$

The force one-form $\Delta^{Y}$ is therefore given by

$$
\Delta^{Y}\left(q^{k}, \dot{q}^{k}\right)=\frac{\partial^{2} L}{\partial \dot{q}^{i} \partial \dot{q}^{j}} Y^{j}\left(q^{k}, \dot{q}^{k}\right) d q^{i}
$$

and the corresponding force field is

$$
F^{Y}=\left(q^{i}, \frac{\partial^{2} L}{\partial \dot{q}^{i} \partial \dot{q}^{j}} Y^{j}\left(q^{k}, \dot{q}^{k}\right)\right) .
$$

Thus, the condition for an integral curve takes the form of the standard EulerLagrange equations with forces:

$$
\frac{d}{d t}\left(\frac{\partial L}{\partial \dot{q}^{i}}\right)-\frac{\partial L}{\partial q^{i}}=F_{i}^{Y}\left(q^{k}, \dot{q}^{k}\right) .
$$

Since the integral Lagrange-d'Alembert principle gives the same equations, it follows that the two principles are equivalent. From now on, we will refer to either one as simply the Lagrange-d'Alembert principle.

Finally, if the force field is given by a Rayleigh dissipation function $R$, then the Euler-Lagrange equations with forcing become:

$$
\frac{d}{d t}\left(\frac{\partial L}{\partial \dot{q}^{i}}\right)-\frac{\partial L}{\partial q^{i}}=-\frac{\partial R}{\partial \dot{q}^{i}}
$$

Combining Corollary $\mathbf{3 . 3}$ with the fact that the differential of $E$ along $Z$ is zero, we find that under the flow of the Euler-Lagrange equations with forcing of Rayleigh dissipation type,

$$
\frac{d}{d t} E(q, v)=F(v) \cdot v=-\mathbb{F} R(q, v) \cdot v<0
$$

\section{Equivariant Dissipation}

In this section we study Lagrangian systems that are invariant under a group action and we will add to them, in the sense of the preceding section, dissipative vector fields that are equivariant. This invariance property will yield dissipative mechanisms that preserve the basic conserved quantities, yet dissipate energy, as we shall see.

Let $G$ be a Lie group that acts on the configuration manifold $Q$ and assume that the lifted action leaves the Lagrangian $L$ invariant. In this case, the fiber derivative 
$\mathbb{F} L: T Q \rightarrow T^{*} Q$ is equivariant with respect to this action on $T Q$ and the dual action on $T^{*} Q$. Evidently, the action $A$, the energy $E$, and the Lagrangian two form $\Omega_{L}$ are all invariant under the action of $G$ on $T Q$. Let $Z$ be the Lagrangian vector field for the Lagrangian $L$, which we assume to be regular. Because of regularity, the vector field $Z$ is also invariant under $G$. If the action is free and proper, so that $(T Q) / G$ is a manifold, then the vector field and its flow $F_{t}$ drop to a vector field $Z^{G}$ and flow $F_{t}^{G}$ on $(T Q) / G$. The determination of this dropped vector field and flow is the subject of Lagrangian reduction (see Marsden and Scheurle [1993a,b]).

Let $\mathbf{J}: T Q \rightarrow \mathfrak{g}^{*}$ be the momentum map associated with the $G$ action, given by

$$
\mathbf{J}\left(v_{q}\right) \cdot \xi=\left\langle\mathbb{F} L\left(v_{q}\right), \xi_{Q}(q)\right\rangle
$$

for $v_{q} \in T_{q} Q$ and for $\xi \in \mathfrak{g}$, where $\xi_{Q}$ denotes the infinitesimal generator for the action on $Q$. The infinitesimal generator for the action on the tangent bundle will be likewise denoted by $\xi_{T Q}$ and for later use, we note the relation $T \tau \circ \xi_{T Q}=\xi_{Q} \circ \tau$. If $v(t)$ denotes an integral curve of the vector field with an equivariant dissipation term $Y$ added, as in the preceding section, and we let $J^{\xi}(v)=\langle\mathbf{J}(v), \xi\rangle$ be the $\xi$-component of the momentum mapping, then we have

$$
\frac{d}{d t} J^{\xi}(v(t))=\mathbf{d} J^{\xi}(v(t)) \cdot Z(c(t))+\mathbf{d} J^{\xi}(v(t)) \cdot Y(v(t))
$$

The first term vanishes by conservation of the momentum map for the Lagrangian vector field $Z$. From (3.10) and the definition of the momentum map, we get

$$
\begin{aligned}
\mathbf{d} J^{\xi}(v) \cdot Y(v) & =\left(\mathbf{i}_{\xi_{T Q}} \Omega_{L}\right)(Y)(v) \\
& =-\left(\mathbf{i}_{Y} \Omega_{L}\right)\left(\xi_{T Q}\right)(v) \\
& =\left\langle F^{Y}(v), T_{v} \tau\left(\xi_{T Q}(v)\right)\right\rangle \\
& =\left\langle F^{Y}(v), \xi_{Q}(\tau(v))\right\rangle
\end{aligned}
$$

and therefore

$$
\frac{d}{d t} J^{\xi}(v(t))=\left\langle F^{Y}, \xi_{Q} \circ \tau\right\rangle(v)
$$

In particular, if $F$ is determined by a Rayleigh dissipation function, we get

$$
\frac{d}{d t} J^{\xi}(v(t))=-\left\langle\mathbb{F} R, \xi_{Q} \circ \tau\right\rangle(v(t)) .
$$

We summarize this discussion as follows.

Proposition 4.1 The momentum map $\mathbf{J}: T Q \rightarrow \mathfrak{g}^{*}$ is conserved under the flow of a $G$-invariant dissipative vector field $Z+Y$ if and only if $\left\langle F^{Y}, \xi_{Q} \circ \tau\right\rangle=0$ for all Lie algebra elements $\xi \in \mathfrak{g}$. If the force field $F^{Y}$ is given by a Rayleigh dissipation function $R: T Q \rightarrow \mathbb{R}$, i.e., $F^{Y}=-\mathbb{F} R$, then this condition becomes $\left\langle\mathbb{F} R, \xi_{Q} \circ \tau\right\rangle=0$ for all $\xi \in \mathfrak{g}$. Moreover, $G$-invariance of $Y$ is equivalent to $G$-equivariance of $\mathbb{F} R$ and if $R$ is $G$-invariant, then $\mathbb{F} R$ is $G$-equivariant. 
We note that equivariance of $\mathbb{F} R$ need not imply invariance of $R$. (Consider, for example, $G=S^{1}$ and $Q=S^{1}$ with $R(\theta, \dot{\theta})=(\dot{\theta})^{2} / 2+f(\theta)$, where $f$ is any non-invariant function of $\theta$ such as $f(\theta)=\sin \theta$.) Also note that if the action of $G$ on $Q$ is transitive, then conservation of $\mathbf{J}$ along the flow of $Z+Y$ implies that the force field $F^{Y}$ vanishes and hence, if $L$ is regular, that $Y$ also vanishes. Thus, in the regular case and for a transitive group action, there is no dissipative vector field preserving the momentum map.

In this paper we shall consider dissipative vector fields for which the flow drops to the reduced spaces. Thus, a first requirement is that $Y$ be a vertical $G$-invariant vector field on $T Q$. A second requirement is that all integral curves $v(t)$ of $Z+Y$ preserve the sets $\mathbf{J}^{-1}(\mathcal{O})$, where $\mathcal{O}$ is an arbitrary coadjoint orbit in $\mathfrak{g}^{*}$. Under these hypotheses the vector field $Z+Y$ induces a vector field $Z^{G}+Y^{G}$ on $(T Q) / G$ that preserves the symplectic leaves of this Poisson manifold, namely all reduced spaces $\mathbf{J}^{-1}(\mathcal{O}) / G$.

The condition that $v(t) \in \mathbf{J}^{-1}(\mathcal{O})$ is equivalent to $\mathbf{J}(v(t)) \in \mathcal{O}$, i.e., to the existence of an element $\eta(t) \in \mathfrak{g}$ such that $d \mathbf{J}(v(t)) / d t=\operatorname{ad}_{\eta(t)}^{*} \mathbf{J}(v(t))$, or

$$
\frac{d J^{\xi}(v(t))}{d t}=J^{[\eta(t), \xi]}(v(t))
$$

for all $\xi \in \mathfrak{g}$. In view of (4.4), we get the following:

Corollary 4.2 The integral curves of the vector field $Z+Y$, for $Y$ a vertical $G$ invariant vector field on $T Q$ and $Z$ the Lagrangian vector field of a $G$-invariant Lagrangian function $L: T Q \rightarrow \mathbb{R}$, preserve the inverse images of the coadjoint orbits in $\mathfrak{g}^{*}$ by the momentum map $\mathbf{J}$ if and only if for each $v \in T Q$ there is some $\eta(v) \in \mathfrak{g}$ such that

$$
\left\langle F^{Y}, \xi_{Q} \circ \tau\right\rangle(v)=J^{[\eta(v), \xi]}(v)
$$

for all $\xi \in \mathfrak{g}$. As before, $F^{Y}$ denotes the force field induced by $Y$.

We will see in section 5 how to construct such force fields in the case $Q=G$. As we mentioned in the introduction, these force fields do not literally come from a Rayleigh dissipation function in the naive sense, but rather come from a Rayleigh dissipation function (the energy itself!) in a more sophisticated sense.

\section{The Euler-Poincaré equations}

If $\mathfrak{g}$ is a Lie algebra and $l: \mathfrak{g} \rightarrow \mathbb{R}$ is a (possibly time dependent) function, the Euler-Poincaré equations for $l$ are the equations

$$
\frac{d}{d t} \frac{\partial l}{\partial \xi}=\operatorname{ad}_{\xi}^{*} \frac{\partial l}{\partial \xi}
$$

These equations include the equations for rigid bodies and fluids, but in the latter case, one must use infinite dimensional Lie algebras. Because of this, we usually

make use of the functional derivative notation and write, eg, $\delta l / \delta \xi$ rather than use 
the partial derivative notation. These equations have a long history, but were first written down for general Lie algebras by Poincaré [1901] (see also Arnold [1988], Chetaev [1961] and Marsden and Ratiu [1994]). These equations are equivalent to the Lie Poisson equations on duals of Lie algebras via the Legendre transformation, as we shall recall below, but apparently Poincaré was unaware of Lie's earlier work. Following Poincaré's fundamental contributions, much confusion seemed to have arisen in the literature and many misconceptions were propagated through the use of terms like "quasicoordinates" etc. We now realize that a good way to derive these equations and to study the associated variational principle is through the methods of Lagrangian reduction.

The general question of reducing variational principles is a complicated one with a mixed history. When a variational principle is reduced, one generally gets a constrained variational principle similar to the so called Lagrange d'Alembert principle for nonholonomic systems. For example, until Marsden and Scheurle [1993b], one cannot even find a clear statement of this principle for the Euler equations for rigid body motion, although one might argue that it is implicit in Poincaré [1901]. For fluid mechanics, it is partly contained in Lin's work on what are commonly called "Lin constraints" (see, for example, Seliger and Whitham [1968]), although a definitive and clear formulation along these lines for fluids and MHD was already given by Newcomb [1962]. We also note that these issues also come up in optimal control and in fact, the methods of Lagrangian reduction can often be used as a substitute for the Pontryagin maximum principle, which focusses on the Hamiltonian side. In particular, some of these ideas are contained in the work of Brockett [1973], who studies the reduction of optimal control problems on compact matrix groups to spheres (adjoint orbits). For the way in which double brackets come into optimal control problems, see Brockett [1994] and for relations with Lagrangian reduction, see Bloch and Crouch [1994].

Below we state the reduction theorem for the general Euler-Poincaré equations. These results were stated in Marsden and Scheurle [1993b], but proofs were given only for the case of matrix groups. Here we give an alternative proof for matrix groups in the text and prove the general result in the appendix. Although many aspects of the general case are not needed for what follows, the proof was instrumental in the development of our ideas in this paper. In particular, it is important in understanding the forced Euler-Poincaré equations.

A key step in the reduction of the Euler-Lagrange equations from the tangent bundle $T G$ of a Lie group $G$ to its Lie algebra $\mathfrak{g}$ is to understand how to drop the variational principle to the quotient space. To do this, we need to characterize variations of curves in $T G$ purely in terms of variations of curves in the Lie algebra $\mathfrak{g}$. The following proposition answers this question.

Proposition 5.1 Let $g: U \subset \mathbb{R}^{2} \rightarrow G$ be a smooth map and denote its partial derivatives by

$$
\xi(t, \varepsilon)=T L_{g(t, \varepsilon)^{-1}}(\partial g(t, \varepsilon) / \partial t)
$$

and

$$
\eta(t, \varepsilon)=T L_{g(t, \varepsilon)^{-1}}(\partial g(t, \varepsilon) / \partial \varepsilon)
$$


Then

$$
\frac{\partial \xi}{\partial \varepsilon}-\frac{\partial \eta}{\partial t}=[\xi, \eta]
$$

Conversely, if $U$ is simply connected and $\xi, \eta: U \rightarrow \mathfrak{g}$ are smooth functions satisfying (5.1) then there exists a smooth function $g: U \rightarrow G$ such that $\xi(t, \varepsilon)=$ $T L_{g(t, \varepsilon)^{-1}}(\partial g(t, \varepsilon) / \partial t)$ and $\eta(t, \varepsilon)=T L_{g(t, \varepsilon)^{-1}}(\partial g(t, \varepsilon) / \partial \varepsilon)$.

We give below the proof of the easy implication for matrix groups only. The converse implication as well as the case for general Lie groups is relegated to the appendix since it is considerably more technical and would disturb the main flow of the paper regarding the dissipation induced instability phenomenon.

Proof of (5.1) for matrix groups When the elements $g$ consist of matrices, we can write

$$
\xi(t, \epsilon)=g(t, \epsilon)^{-1} \frac{\partial g(t, \epsilon)}{\partial t}
$$

and

$$
\eta(t, \epsilon)=g(t, \epsilon)^{-1} \frac{\partial g(t, \epsilon)}{\partial \epsilon} .
$$

Differentiating these expressions using the product rule and equality of mixed partial derivatives gives

$$
\begin{aligned}
\frac{\partial \xi}{\partial \varepsilon}-\frac{\partial \eta}{\partial t} & =-g^{-1} \frac{\partial g}{\partial \epsilon} g^{-1} \frac{\partial g}{\partial t}+g^{-1} \frac{\partial^{2} g}{\partial \epsilon \partial t}+g^{-1} \frac{\partial g}{\partial t} g^{-1} \frac{\partial g}{\partial t}-g^{-1} \frac{\partial^{2} g}{\partial t \partial \epsilon} \\
& =\xi \eta-\eta \xi=[\xi, \eta]
\end{aligned}
$$

Next, we turn to the formulation of the Euler-Poincaré equations and the reduced variational principle.

Theorem 5.2 Let $G$ be a Lie group and $L: T G \rightarrow \mathbb{R}$ a left invariant Lagrangian. Let $l: \mathfrak{g} \rightarrow \mathbb{R}$ be its restriction to the tangent space at the identity. For a curve $g(t) \in G$, let $\xi(t)=g(t)^{-1} \cdot \dot{g}(t) ;$ i.e., $\xi(t)=T_{g(t)} L_{g(t)^{-1}} \dot{g}(t)$. Then the following are equivalent:

i $g(t)$ satisfies the Euler-Lagrange equations for $L$ on $G$.

ii The variational principle

$$
\delta \int_{a}^{b} L(g(t), \dot{g}(t)) d t=0
$$

holds, for variations with fixed endpoints.

iii The Euler-Poincaré equations hold

$$
\frac{d}{d t} \frac{\delta l}{\delta \xi}=\operatorname{ad}_{\xi}^{*} \frac{\delta l}{\delta \xi}
$$


iv The variational principle

$$
\delta \int_{a}^{b} l(\xi(t)) d t=0
$$

holds on $\mathfrak{g}$, using variations of the form

$$
\delta \xi=\dot{\eta}+[\xi, \eta]
$$

where $\eta$ vanishes at the endpoints.

In coordinates, the Euler-Poincaré equations read as follows

$$
\frac{d}{d t} \frac{\partial l}{\partial \xi^{d}}=C_{a d}^{b} \frac{\partial l}{\partial \xi^{b}} \xi^{a}
$$

where $C_{a d}^{b}$ are the structure constants of $\mathfrak{g}$ relative to a given basis and $\xi^{a}$ are the components of $\xi$ relative to this basis.

Proof The equivalence of $\mathbf{i}$ and $\mathbf{i i}$ holds for any configuration manifold $Q$ and so, in particular, for $Q=G$.

Next, we prove that ii and iv are equivalent. First, note that $l: \mathfrak{g} \rightarrow \mathbb{R}$ determines uniquely a function $L: T G \rightarrow \mathbb{R}$ by left translation of the argument and conversely. Thus, the equivalence of $\mathbf{i i}$ and iv comes down to proving that all variations $\delta g(t) \in T G$ of $g(t)$ with fixed endpoints induce and are induced by variations $\delta \xi(t)$ of $\xi(t)$ of the form $\delta \xi=\dot{\eta}+[\xi, \eta]$, where $\eta(t)$ vanishes at the endpoints. This, however, is precisely the content of Proposition 5.1.

To complete the proof, we show the equivalence of iii and iv. Indeed, using the definitions and integrating by parts,

$$
\begin{aligned}
\delta \int l(\xi) d t & =\int \frac{\delta l}{\delta \xi} \delta \xi d t \\
& =\int \frac{\delta l}{\delta \xi}\left(\dot{\eta}+\operatorname{ad}_{\xi} \eta\right) d t \\
& =\int\left[-\frac{d}{d t}\left(\frac{\delta l}{\delta \xi}\right)+\operatorname{ad}_{\xi}^{*} \frac{\delta l}{\delta \xi}\right] \eta d t
\end{aligned}
$$

and so the result follows.

Since the Euler-Lagrange and Hamilton equations on $T Q$ and $T^{*} Q$ are equivalent if the fiber derivative of $L$ is a diffeomorphism from $T Q$ to $T^{*} Q$, it follows that the Lie-Poisson and Euler-Poincaré equations are also equivalent under similar hypotheses. To see this directly, we make the following Legendre transformation from $\mathfrak{g}$ to $\mathfrak{g}^{*}$ :

$$
\mu=\frac{\delta l}{\delta \xi}, \quad h(\mu)=\langle\mu, \xi\rangle-l(\xi)
$$

and assume that $\xi \mapsto \mu$ is a diffeomorphism. Note that

$$
\frac{\delta h}{\delta \mu}=\xi+\left\langle\mu, \frac{\delta \xi}{\delta \mu}\right\rangle-\left\langle\frac{\delta l}{\delta \xi}, \frac{\delta \xi}{\delta \mu}\right\rangle=\xi
$$


and so it is now clear that the Euler-Poincaré equations are equivalent to the LiePoisson equations on $\mathfrak{g}^{*}$, namely

$$
\frac{d \mu}{d t}=\operatorname{ad}_{\delta h / \delta \mu}^{*} \mu
$$

which is equivalent to $\dot{F}=\{F, h\}$ relative to the Lie-Poisson bracket (see Marsden [1992] for more information and references).

As an example, let us consider the free rigid body equations. Here $G=S O(3), \mathfrak{g}=$ $\left(\mathbb{R}^{3}, \times\right)$ and $l(\Omega)=(1 / 2) \mathbb{I} \Omega \cdot \Omega$, where $\mathbb{I}=\operatorname{diag}\left(I_{1}, I_{2}, I_{3}\right)$. For an arbitrary vector $\delta \Omega \in \mathbb{R}^{3}$ we have

$$
\left\langle\frac{\delta l}{\delta \Omega}, \delta \Omega\right\rangle=\mathbf{D} l(\Omega) \cdot \delta \Omega=\mathbb{I} \Omega \cdot \delta \Omega
$$

so that identifying $\mathbb{R}^{3}$ with itself relative to the dot product we get $\delta l / \delta \Omega=\mathbb{I} \Omega$. Moreover,

$$
\left\langle\operatorname{ad}_{\Omega}^{*} \frac{\delta l}{\delta \Omega}, \delta \Omega\right\rangle=\left\langle\frac{\delta l}{\delta \Omega}, \Omega \times \delta \Omega\right\rangle=\frac{\delta l}{\delta \Omega} \cdot(\Omega \times \delta \Omega)=(\mathbb{I} \Omega \times \Omega) \cdot \delta \Omega,
$$

so that

$$
\operatorname{ad}_{\Omega}^{*} \frac{\delta l}{\delta \Omega}=\mathbb{I} \Omega \times \Omega
$$

and therefore the Euler-Poincaré equations are

$$
\mathbb{I} \dot{\Omega}=\mathbb{I} \Omega \times \Omega .
$$

which are the classical Euler equations in the body representation.

\section{Dissipation for Euler-Poincaré and Lie-Poisson Equa- tions}

Now we are ready to synthesize our discussions of forces and of the Euler-Poincaré equations and to transfer this forcing to the Lie-Poisson equations by means of the Legendre transform. We begin with a formulation of the Lagrange-d'Alembert principle.

Theorem 6.1 Let $G$ be a Lie group, $L: T G \rightarrow \mathbb{R}$ a left invariant Lagrangian, and $F: T G \rightarrow T^{*} G$ a force field equivariant relative to the canonical left actions of $G$ on $T G$ and $T^{*} G$ respectively. Let $l: \mathfrak{g} \rightarrow \mathbb{R}$ and $f: \mathfrak{g} \rightarrow \mathfrak{g}^{*}$ be the restriction of $L$ and $F$ to $T_{e} G=\mathfrak{g}$. For a curve $g(t) \in G$, let $\xi(t)=T_{g(t)} L_{g(t)^{-1}} \dot{g}(t)$. Then the following are equivalent:

i $g(t)$ satisfies the Euler-Lagrange equations with forcing for $L$ on $G$.

ii The integral Lagrange-d'Alembert principle

$$
\delta \int_{a}^{b} L(g(t), \dot{g}(t)) d t=\int_{a}^{b} F(g(t), \dot{g}(t)) \cdot \delta g(t) d t
$$

holds for all variations $\delta g(t)$ with fixed endpoints. 
iii The Euler-Poincaré equations with forcing are valid:

$$
\frac{d}{d t} \mathbf{D} l(\xi)-\operatorname{ad}_{\xi}^{*} \mathbf{D} l(\xi)=f(\xi)
$$

iv The variational principle

$$
\delta \int_{a}^{b} l(\xi(t)) d t=\int_{a}^{b} f(\xi(t)) \cdot \delta \xi(t) d t
$$

holds on $\mathfrak{g}$, using variations of the form

$$
\delta \xi=\dot{\eta}+[\xi, \eta]
$$

where $\eta$ vanishes at the endpoints.

Proof We have already seen that $\mathbf{i}$ and ii are equivalent for any configuration manifold $Q$ in section 2 . The equivalence of $\mathbf{i i}$ and iv and of iii and iv repeats the proof of Theorem $\mathbf{4 . 5}$.

The Euler-Poincaré equations with forcing have the following expression in local coordinates

$$
\frac{d}{d t} \frac{\partial l}{\partial \xi^{a}}-C_{b a}^{d} \frac{\partial l}{\partial \xi^{d}}=f_{a}
$$

where $C_{b a}^{d}$ are the structure constants of the Lie algebra $\mathfrak{g}$.

The condition that the integral curves of the dissipative vector field preserve the inverse images of coadjoint orbits by the momentum map and hence the integral curves of (6.2) preserve the coadjoint orbits of $\mathfrak{g}^{*}$ is given by (4.6). Since $\xi_{G}(g)=$ $T_{e} R_{g}(\xi)$ and $\mathbf{J}\left(v_{g}\right)=T_{e}^{*} R_{g} \mathbb{F} L\left(v_{g}\right)$, we get

$$
\left\langle F, \xi_{G} \circ \tau\right\rangle\left(v_{g}\right)=\left\langle F\left(v_{g}\right), T_{e} R_{g}(\xi)\right\rangle=T_{e}^{*} R_{g} F\left(v_{g}\right) \cdot \xi
$$

and

$$
\begin{aligned}
J^{\left[\eta\left(v_{g}\right), \xi\right]}\left(v_{g}\right) & =T_{e}^{*} R_{g} \mathbb{F} L\left(v_{g}\right) \cdot\left[\eta\left(v_{g}\right), \eta\right] \\
& =\left(\operatorname{ad}_{\eta\left(v_{g}\right)}^{*} \circ T_{e}^{*} R_{g} \circ \mathbb{F} L\right)\left(v_{g}\right) \cdot \xi .
\end{aligned}
$$

Since $F$ and $\mathbb{F} L$ are equivariant,

$$
T_{e}^{*} R_{g} F\left(v_{g}\right)=\operatorname{Ad}_{g^{-1}}^{*} F\left(T_{g} L_{g^{-1}} v_{g}\right),
$$

and

$$
\left(\operatorname{ad}_{\eta\left(v_{g}\right)}^{*} \circ T_{e}^{*} R_{g} \circ \mathbb{F} L\right)\left(v_{g}\right)=\left(\operatorname{ad}_{\eta\left(v_{g}\right)}^{*} \circ \operatorname{Ad}_{g^{-1}}^{*} \circ \mathbb{F} L\right)\left(T_{g} L_{g^{-1}} v_{g}\right) .
$$

However, $\operatorname{Ad}_{g^{-1}} \circ \operatorname{ad}_{\eta\left(v_{g}\right)}=\operatorname{ad}_{\operatorname{Ad}_{g^{-1}} \eta\left(v_{g}\right)} \circ \operatorname{Ad}_{g^{-1}}$, and thus we get

$$
J^{\left[\eta\left(v_{g}\right), \xi\right]}\left(v_{g}\right)=\left(\operatorname{Ad}_{g^{-1}}^{*} \circ \operatorname{ad}_{\mathrm{Ad}_{g^{-1}} \eta\left(v_{g}\right)}^{*} \circ \mathbb{F} L\right)\left(T_{g} L_{g^{-1}} v_{g}\right)
$$


and the identity (4.6) thus becomes

$$
F\left(T_{g} L_{g^{-1}} v_{g}\right)=\left(\operatorname{ad}_{\mathrm{Ad}_{g^{-1}} \eta\left(v_{g}\right)}^{*} \circ \mathbb{F} L\right)\left(T_{g} L_{g^{-1}} v_{g}\right) .
$$

Letting $\zeta=T_{g} L_{g^{-1}} v_{g}$, this becomes

$$
f(\zeta)=\operatorname{ad}_{\mathrm{Ad}_{g^{-1}} \eta\left(v_{g}\right)} \mathbf{D} l(\zeta) .
$$

The left hand side is independent of $g$ and thus the right hand side must be also $g$-independent. Thus taking $g=e$, the criterion (4.6) becomes: for every $\zeta \in \mathfrak{g}$, there is some $\eta(\zeta) \in \mathfrak{g}$ such that

$$
f(\zeta)=\operatorname{ad}_{\eta(\zeta)}^{*} \mathbf{D} l(\zeta)
$$

In other words, the force field $f$ (and hence $F$ ) is completely determined by an arbitrary map $\eta: \mathfrak{g} \rightarrow \mathfrak{g}$ via formula (6.6) and we conclude the following.

Corollary 6.2 The solutions of the Euler-Poincaré equations with forcing (6.2) preserve the coadjoint orbits of $\mathfrak{g}^{*}$ provided the force field $f$ is given by (6.6) for some smooth map $\eta: \mathfrak{g} \rightarrow \mathfrak{g}$.

Next, we want to restore Rayleigh dissipation functions as much as possible. As we have mentioned in the introduction, the force field terms we want for the rigid body cannot literally come from such a function. Relaxing this slightly, we will ask that they be gradient relative to a metric on the orbit.

We begin with transforming the Euler-Poincaré equations with the forcing by means of the Legendre transform, namely

$$
\mu=\mathbf{D} l(\xi), \quad h(\mu)=\langle\mu, \xi\rangle-l(\xi) .
$$

Then the functional derivative of the Hamiltonian $h: \mathfrak{g}^{*} \rightarrow \mathbb{R}$ equals $\delta h / \delta \mu=\xi$ and (6.2) with the force field term (6.6) becomes

$$
\frac{d \mu}{d t}-\operatorname{ad}_{\delta h / \delta \mu}^{*} \mu=-\operatorname{ad}_{\eta(\mu)}^{*} \mu
$$

where $\eta: \mathfrak{g}^{*} \rightarrow \mathfrak{g}$. (We have changed $\eta$ to $-\eta$ for later convenience.) The requirement on the map $\eta$ is that the right hand side of (6.8) be a gradient relative to a certain metric on the orbit.

This Riemannian metric is usually defined on adjoint orbits of semi-simple compact Lie algebras in the following manner. The negative of the Killing form defines by left translation a left-invariant metric on the group $G$. Given the adjoint orbit $\mathcal{O}$ containing the element $\mu \in \mathfrak{g}$, it is diffeomorphic to $G / G_{\mu}$, where $G_{\mu}$ is the isotropy subgroup of the adjoint action at $\mu$. The Riemannian metric drops to the quotient $G / G_{\mu}$ and therefore the above mentioned diffeomorphism pushes it forward to a Riemannian metric on $\mathcal{O}$, called the normal metric. In general, this metric is not Kähler but, due to bi-invariance of the Killing form, it is $G$-invariant. An explicit formula for this metric is as follows. If $[\mu, \eta],[\mu, \zeta] \in T_{\mu} \mathcal{O}$, their inner product is

$$
\langle[\mu, \eta],[\mu, \zeta]\rangle_{N}=-\kappa\left(\eta^{\mu}, \zeta^{\mu}\right),
$$


where $\kappa$ is the Killing form of $\mathfrak{g}$ and $\eta^{\mu}, \zeta^{\mu}$ are the $\mathfrak{g}^{\mu}$-components of $\eta$ and $\zeta$ respectively in the direct sum orthogonal decomposition

$$
\mathfrak{g}=\mathfrak{g}_{\mu} \oplus \mathfrak{g}^{\mu}
$$

for $\mathfrak{g}_{\mu}=\operatorname{ker}\left(\operatorname{ad}_{\mu}\right), \mathfrak{g}^{\mu}=\operatorname{range}\left(\operatorname{ad}_{\mu}\right)$.

To generalize this metric to coadjoint orbits of the dual $\mathfrak{g}^{*}$ of a general Lie algebra $\mathfrak{g}$, we introduce a symmetric positive definite bilinear form $\tilde{\Gamma}: \mathfrak{g}^{*} \times \mathfrak{g}^{*} \rightarrow \mathbb{R}$. We also refer to Brockett [1993] for a related generalization in the compact case.

Denote by $\Gamma: \mathfrak{g}^{*} \rightarrow \mathfrak{g}$ the induced map given by $\tilde{\Gamma}(\alpha, \beta)=\langle\beta, \Gamma \alpha\rangle$ for all $\alpha, \beta \in \mathfrak{g}^{*}$, where $\langle\rangle:, \mathfrak{g}^{*} \times \mathfrak{g} \rightarrow \mathbb{R}$ denotes the pairing between $\mathfrak{g}^{*}$ and $\mathfrak{g}$. Symmetry of $\tilde{\Gamma}$ is equivalent to symmetry of $\Gamma$, i.e. $\Gamma^{*}=\Gamma$. We introduce the following new inner product on $\mathfrak{g}$ :

$$
\langle\xi, \eta\rangle_{\Gamma^{-1}}=\left\langle\Gamma^{-1} \eta, \xi\right\rangle
$$

for all $\xi, \eta \in \mathfrak{g}$, and call it the $\Gamma^{-1}$-inner product. Let $\mathfrak{g}_{\mu}$ denote the coadjoint isotropy subalgebra of $\mu$, i.e. the kernel of the map $\xi \mapsto \operatorname{ad}_{\xi}^{*} \mu$, and denote by $\mathfrak{g}^{\mu}$ its orthogonal complement relative to the $\Gamma^{-1}$-inner product. For an element $\xi \in \mathfrak{g}$ we denote by $\xi_{\mu}$ and $\xi^{\mu}$ the components of $\xi$ in the orthogonal direct sum decomposition $\mathfrak{g}=\mathfrak{g}_{\mu} \oplus \mathfrak{g}^{\mu}$.

Let $C$ be a positive Casimir function on $\mathfrak{g}^{*}$ and let $\mathcal{O}_{\mu_{0}}$ be the coadjoint orbit through $\mu_{0} \in \mathfrak{g}^{*}$. If $\mu \in \mathcal{O}_{\mu_{0}}$, then $\operatorname{ad}_{\xi}^{*} \mu \in T_{\mu} \mathcal{O}_{\mu_{0}}$ and we define the $\left(C, \Gamma^{-1}\right)$-normal metric on $\mathcal{O}_{\mu_{0}}$ by

$$
\left\langle\operatorname{ad}_{\xi}^{*} \mu, \operatorname{ad}_{\eta}^{*} \mu\right\rangle_{N}=C(\mu)\left\langle\eta^{\mu}, \xi^{\mu}\right\rangle_{\Gamma^{-1}}=C(\mu)\left\langle\Gamma^{-1} \eta^{\mu}, \xi^{\mu}\right\rangle .
$$

We will regard $C$ and $\Gamma$ as fixed in the following discussion and just refer to this metric as the normal metric. Let $k: \mathfrak{g}^{*} \rightarrow \mathbb{R}$ be a smooth function. We will compute the gradient vector field of $k \mid \mathcal{O}_{\mu_{0}}$ relative to this normal metric. For this purpose denote by $\delta k / \delta \mu \in \mathfrak{g}$ the functional derivative of $k$ at $\mu$ and by $\operatorname{grad} k(\mu)$ the gradient of $k \mid \mathcal{O}_{\mu_{0}}$. Since $\operatorname{grad} k(\mu) \in T_{\mu} \mathcal{O}_{\mu_{0}}$, we can write $\operatorname{grad} k(\mu)=\operatorname{ad}_{\eta}^{*} \mu$ for some $\eta \in \mathfrak{g}$. Since $\xi_{\mu}$ and $\eta^{\mu}$ are orthogonal in the $\Gamma^{-1}$-inner product, we get

$$
\begin{aligned}
-\left\langle\operatorname{ad}_{\delta k / \delta \mu}^{*} \mu, \xi\right\rangle & =\left\langle\mu,\left[\xi, \frac{\delta k}{\delta \mu}\right]\right\rangle=\left\langle\operatorname{ad}_{\xi}^{*} \mu, \frac{\delta k}{\delta \mu}\right\rangle=\mathbf{D} k(\mu) \cdot \operatorname{ad}_{\xi}^{*} \mu \\
& =\left\langle\operatorname{grad} k(\mu), \operatorname{ad}_{\xi}^{*} \mu\right\rangle_{N}=\left\langle\operatorname{ad}_{\eta}^{*} \mu, \operatorname{ad}_{\xi}^{*} \mu\right\rangle_{N} \\
& =C(\mu)\left\langle\Gamma^{-1} \xi^{\mu}, \eta^{\mu}\right\rangle=C(\mu)\left\langle\Gamma^{-1}\left(\xi^{\mu}+\xi_{\mu}\right), \eta^{\mu}\right\rangle \\
& =C(\mu)\left\langle\Gamma^{-1} \xi, \eta^{\mu}\right\rangle=C(\mu)\left\langle\Gamma^{-1} \eta^{\mu}, \xi\right\rangle
\end{aligned}
$$

for any $\xi \in \mathfrak{g}$. Therefore $C(\mu) \Gamma^{-1} \eta^{\mu}=-\operatorname{ad}_{\delta k / \delta \mu}^{*} \mu$, or

$$
\eta^{\mu}=-\frac{1}{C(\mu)} \Gamma\left(\operatorname{ad}_{\delta k / \delta \mu}^{*} \mu\right)
$$

Thus $\operatorname{grad} k(\mu)=\operatorname{ad}_{\eta}^{*} \mu=\operatorname{ad}_{\eta^{\mu}}^{*} \mu=-(1 / C(\mu)) \operatorname{ad}_{\Gamma\left(\operatorname{ad}_{\delta k / \delta \mu}^{*} \mu\right)}^{*} \mu$ and the equation of motion for the gradient vector field of $k \mid \mathcal{O}_{\mu_{0}}$ relative to the normal metric on the coadjoint orbit $\mathcal{O}_{\mu_{0}}$ is

$$
\frac{d \mu}{d t}=-\frac{1}{C(\mu)} \operatorname{ad}_{\Gamma\left(\operatorname{ad}_{\delta k / \delta \mu}^{*} \mu\right)}^{*} \mu .
$$


Therefore, in (6.8), we will choose $\eta(\mu)=-(1 / C(\mu)) \Gamma\left(\operatorname{ad}_{\delta k / \delta \mu}^{*}\right) \mu$ and the LiePoisson equations with forcing (6.8) become

$$
\frac{d \mu}{d t}-\operatorname{ad}_{\delta h / \delta \mu}^{*} \mu=\frac{1}{C(\mu)} \operatorname{ad}_{\Gamma\left(\operatorname{ad}_{\delta k / \delta \mu}^{*} \mu\right)}^{*} \mu .
$$

The term we added is the negative of the $\left(C, \Gamma^{-1}\right)$-normal metric gradient. If $\mathfrak{g}$ is a compact Lie algebra, let $\langle\cdot, \cdot\rangle$ be a bi-invariant inner product on $\mathfrak{g}$; if $\mathfrak{g}$ is also semisimple we could let $\langle\cdot, \cdot\rangle=-\kappa(\cdot, \cdot)$, where $-\kappa(\cdot, \cdot)$ is the Killing form. This inner product identifies $\mathfrak{g}$ with its dual, coadjoint orbits with adjoint orbits, so that $\operatorname{ad}_{\xi}^{*} \mu=[\mu, \xi]$, and $\delta k / \delta \mu=\nabla k(\mu)$, where $\nabla k(\mu)$ is the gradient of $k$ on $\mathfrak{g}$ at $\mu$ relative to the bi-invariant inner product $\langle\cdot, \cdot\rangle$. The formula for the gradient vector field on the adjoint orbit $\mathcal{O}_{\mu_{0}}$ becomes

$$
\frac{d \mu}{d t}=-\frac{1}{C(\mu)}[\mu, \Gamma([\mu, \nabla k(\mu)]]
$$

where $\Gamma: \mathfrak{g} \rightarrow \mathfrak{g}$ defines the symmetric positive definite bilinear form $(\xi, \eta) \mapsto\langle\Gamma \xi, \eta\rangle$. Thus, in this case the Lie-Poisson equations with forcing become

$$
\frac{d \mu}{d t}=-[\nabla h(\mu), \mu]+\frac{1}{C(\mu)}[\mu, \Gamma([\mu, \nabla k(\mu)])] .
$$

Taking $C(\mu)=1$ and $\Gamma$ to be the identity, the dissipative term in (6.11) is in Brockett double bracket form. Note again that on the right-hand side, the double bracket is the negative of the usual normal metric gradient.

For example, if $\mathfrak{g}=\mathbb{R}^{3}$, we claim that the normal metric on two tangent vectors $v, w$ tangent to the sphere of radius $c$ is given by

$$
\langle v, w\rangle_{N}=\frac{1}{c^{2}}\langle v, w\rangle
$$

where the inner product on the right hand side is the standard inner product in $\mathbb{R}^{3}$. To see this, write $v=M \times X$ and $w=M \times Y$ and use the definition of the normal metric to give

$$
\langle M \times X, M \times Y\rangle_{N}(M)=\left\langle X^{M}, Y^{M}\right\rangle,
$$

where $X^{M}$ is the tangential component of $X$ to the sphere. But by (2.4) we see that

$$
X^{M}=-\frac{1}{c^{2}} M \times(M \times X) .
$$

Substitution gives

$$
\left\langle X^{M}, Y^{M}\right\rangle=\frac{1}{c^{4}}\langle M,(M \times X) \times(M \times(M \times Y))\rangle .
$$

Now using the vector identity for triple cross products (the bac cab rule) we get the stated result. 
Let us return to the general case. The condition that the forcing terms be dissipative is $d h / d t<0$ (see $\S 3$ ). This will impose conditions on the choice of the function $k: \mathfrak{g}^{*} \rightarrow \mathbb{R}$. We have

$$
\begin{aligned}
\frac{d}{d t} h(\mu(t)) & =\left\langle\dot{\mu}(t), \frac{\delta h}{\delta \mu}\right\rangle \\
& =\frac{1}{C(\mu)}\left\langle\operatorname{ad}_{\Gamma\left(\operatorname{ad}_{\delta k / \delta \mu}^{*} \mu\right)}^{*}, \frac{\delta h}{\delta \mu}\right\rangle \\
& =\frac{1}{C(\mu)}\left\langle\mu,\left[\Gamma\left(\operatorname{ad}_{\delta k / \delta \mu}^{*} \mu\right), \frac{\delta h}{\delta \mu}\right]\right\rangle \\
& =-\frac{1}{C(\mu)}\left\langle\operatorname{ad}_{\delta h / \delta \mu}^{*} \mu, \Gamma\left(\operatorname{ad}_{\delta k / \delta \mu}^{*} \mu\right)\right\rangle \\
& =-\frac{1}{C(\mu)} \tilde{\Gamma}\left(\operatorname{ad}_{\delta k / \delta \mu}^{*} \mu, \operatorname{ad}_{\delta h / \delta \mu}^{*} \mu\right) .
\end{aligned}
$$

Thus, since $\tilde{\Gamma}$ is positive definite and $C$ is positive, the choice $k=h$ will render $d h / d t<0$.

\section{The Lie-Poisson Instability Theorem}

We will now prove an instability theorem in the Lie-Poisson context. However, with little added effort, we can prove a somewhat more general theorem for dissipative systems on Poisson manifolds suggested by the constructions we have given for LiePoisson systems and by the work of Vallis, Carnevale, and Young [1989].

We assume that we are given a Poisson manifold $(P,\{\}$,$) with Poisson tensor$ denoted by $\Lambda$, so that at each point $z \in P$, we have $\Lambda_{z}: T_{z}^{*} P \rightarrow T_{z} P$ given by $\Lambda(\mathbf{d} H)=X_{H}$, i.e. $\langle\mathbf{d} F, \Lambda(\mathbf{d} H)\rangle=\{F, H\}$. By skew-symmetry of the Poisson bracket we have $\Lambda^{*}=-\Lambda$. We also assume that there is a Riemannian metric $\alpha$ defined on each symplectic leaf of $P$. We will use the same notation $\alpha_{z}$ for the induced map $T_{z} S \rightarrow T_{z}^{*} S$, where $S$ is the symplectic leaf through $z$. For a Hamiltonian $H: P \rightarrow \mathbb{R}$ we will consider perturbations of the Hamiltonian vector field $X_{H}$ of the form

$$
\frac{d z}{d t}=\Lambda_{z} \mathbf{d} H(z)+\Lambda_{z} \alpha_{z} \Lambda_{z} \mathbf{d} H(z) .
$$

The second term on the right hand side defines a vector field equivalently given by $\dot{F}=-\{\{F, H\}\}$ for any $F: P \rightarrow \mathbb{R}$, where

$$
\{\{F, H\}\}=-\langle\mathbf{d} F, \Lambda \alpha \Lambda \mathbf{d} H\rangle=\alpha\left(X_{F}, X_{H}\right) .
$$

Thus the full equations can be written as

$$
\dot{F}=\{F, H\}-\{\{F, H\}\}
$$

for any $F: P \rightarrow \mathbb{R}$. 
As an example, take $P=\mathfrak{g}^{*}$ and

$$
\alpha_{\mu}\left(\operatorname{ad}_{\xi}^{*} \mu, \operatorname{ad}_{\eta}^{*} \mu\right)=\frac{1}{C(\mu)} \tilde{\Gamma}\left(\operatorname{ad}_{\xi}^{*} \mu, \operatorname{ad}_{\eta}^{*} \mu\right)
$$

This formula defines on each coadjoint orbit the induced metric given by $\tilde{\Gamma}$, up to the factor $1 / C(\mu)$. For $f, h: \mathfrak{g}^{*} \rightarrow \mathbb{R}$ we get

$$
\begin{aligned}
\left\langle\mathbf{d} f(\mu), \Lambda_{\mu} \alpha_{\mu} \Lambda_{\mu} \mathbf{d} h(\mu)\right\rangle & =-\alpha_{\mu}\left(X_{f}(\mu), X_{h}(\mu)\right) \\
& =-\alpha_{\mu}\left(\operatorname{ad}_{\delta f / \delta \mu}^{*} \mu, \operatorname{ad}_{\delta h / \delta \mu}^{*} \mu\right) \\
& =-\frac{1}{C(\mu)} \tilde{\Gamma}\left(\operatorname{ad}_{\delta f / \delta \mu}^{*} \mu, \operatorname{ad}_{\delta h / \delta \mu}^{*} \mu\right) \\
& =-\frac{1}{C(\mu)}\left\langle\operatorname{ad}_{\delta f / \delta \mu}^{*} \mu, \Gamma\left(\operatorname{ad}_{\delta h / \delta \mu}^{*} \mu\right)\right\rangle \\
& =\frac{1}{C(\mu)}\left\langle\operatorname{ad}_{\Gamma\left(\operatorname{ad}_{\delta h / \delta \mu}^{*} \mu\right)}^{*} \mu, \delta f / \delta \mu\right\rangle \\
& \left.=\left\langle\mathbf{d} f(\mu), \frac{1}{C(\mu)} \operatorname{ad}_{\Gamma\left(\operatorname{ad}_{\delta h / \delta \mu}^{*} \mu\right)}^{*}\right\rangle\right\rangle
\end{aligned}
$$

so that

$$
\Lambda_{\mu} \alpha_{\mu} \Lambda_{\mu} \mathbf{d} h(\mu)=\frac{1}{C(\mu)} \operatorname{ad}_{\Gamma\left(\operatorname{ad}_{\delta h / \delta \mu}^{*} \mu\right)}^{*} \mu
$$

which coincides with the right hand side of equation (6.10), i.e., it is minus the $\left(C, \Gamma^{-1}\right)$-normal metric gradient. Therefore, $-\Lambda \alpha \Lambda$ is the $\left(C, \Gamma^{-1}\right)$-normal metric. Thus the dissipative term considered in the previous section is exactly of this form. The symmetric bracket is hence in this case equal to

$$
\{\{f, h\}\}(\mu)=\frac{1}{C(\mu)} \tilde{\Gamma}\left(\operatorname{ad}_{\delta f / \delta \mu}^{*} \mu, \operatorname{ad}_{\delta h / \delta \mu}^{*} \mu\right) .
$$

It is also interesting to note that this symmetric bracket is the Beltrami bracket given by the normal metric. The Beltrami bracket of two functions on a Riemannian manifold is the inner product of the gradients of the two functions relative to this metric (see Crouch [1981] and references therein). In our case, if $f: \mathfrak{g}^{*} \rightarrow \mathbb{R}$ we saw in the previous section that the gradient of $f$ in the normal metric on the coadjoint orbit has the expression

$$
\operatorname{grad} f(\mu)=-\frac{1}{C(\mu)} \operatorname{ad}_{\Gamma\left(\operatorname{ad}_{\delta f / \delta \mu}^{*} \mu\right)}^{*} \mu .
$$

Since $\mathfrak{g}_{\mu}$ and $\mathfrak{g}^{\mu}$ are orthogonal in the $\Gamma^{-1}$-inner product, we get

$$
\begin{aligned}
\langle\operatorname{grad} f(\mu), \operatorname{grad} h(\mu)\rangle_{N} & =\frac{1}{C(\mu)}\left\langle\left[\Gamma\left(\operatorname{ad}_{\delta f / \delta \mu}^{*} \mu\right)\right]^{\mu},\left[\Gamma\left(\operatorname{ad}_{\delta h / \delta \mu}^{*} \mu\right)\right]^{\mu}\right\rangle_{\Gamma^{-1}} \\
& =\frac{1}{C(\mu)}\left\langle\operatorname{ad}_{\delta f / \delta \mu}^{*} \mu,\left[\Gamma\left(\operatorname{ad}_{\delta h / \delta \mu}^{*} \mu\right)\right]^{\mu}\right\rangle
\end{aligned}
$$


Denoting $\Gamma\left(\operatorname{ad}_{\delta h / \delta \mu}^{*} \mu\right)=\zeta$, this expression equals

$$
\begin{aligned}
\frac{1}{C(\mu)}\left\langle\operatorname{ad}_{\delta f / \delta \mu}^{*} \mu, \zeta^{\mu}\right\rangle & =-\frac{1}{C(\mu)}\left\langle\operatorname{ad}_{\zeta^{\mu}}^{*} \mu, \frac{\delta f}{\delta \mu}\right\rangle \\
& =-\frac{1}{C(\mu)}\left\langle\operatorname{ad}_{\zeta}^{*} \mu, \frac{\delta f}{\delta \mu}\right\rangle \\
& =\frac{1}{C(\mu)}\left\langle\operatorname{ad}_{\delta f / \delta \mu}^{*} \mu, \zeta\right\rangle \\
& =\frac{1}{C(\mu)} \tilde{\Gamma}\left(\operatorname{ad}_{\delta f / \delta \mu}^{*} \mu, \operatorname{ad}_{\delta h / \delta \mu}^{*} \mu\right) \\
& =\{\{f, h\}\}(\mu) .
\end{aligned}
$$

Let us now return to the general case. An important point is that the added dissipative terms of the above form do not destroy the equilibrium. In other words:

Proposition 7.1 If $z_{e}$ is an equilibrium for a Hamiltonian system with Hamiltonian $H$ on a Poisson manifold, then it is also an equilibrium for the system with added dissipative term of the form $\Lambda \alpha \Lambda d H$ as above, or of double bracket form on the dual of a Lie algebra.

Proof An equilibrium $z_{e}$ is characterized by the fact that $X_{H}\left(z_{e}\right)=0$ and the added term is $\Lambda \alpha X_{H}(z)$. In the case of duals of Lie algebras, this can be said this way: the added dissipation does not destroy a given relative equilibrium because it is the gradient of the Hamiltonian on the orbit relative to the normal metric, and the differential of the Hamiltonian restricted to the orbit is zero at a relative equilibrium.

Theorem 7.2 Assume that $z_{e}$ is an equilibrium of a Hamiltonian system on a Poisson manifold (or, specifically, on the dual of a Lie algebra with the Lie Poisson bracket). Assume that the second variation of the Hamiltonian restricted to the symplectic leaf $S\left(z_{e}\right)$ (or coadjoint orbit in the case of the dual of a Lie algebra) through $z_{e}$ is nonsingular but indefinite. Then with a dissipative term of the form $\Lambda \alpha X_{H}(z)$ described above added to the equations, the equilibrium becomes nonlinearly unstable; if the dissipation is small, it is, in addition, spectrally unstable (and hence exponentially unstable) on the leaf.

Proof As is well known and easily verified (see, for example, Marsden, Ratiu and Raugel [1991]), the second variation of the Hamiltonian in the space tangent to the leaf (coadjoint orbit) generates the linearized equations (restricted to the leaf or coadjoint orbit). With dissipation added, we look at the equation

$$
\dot{H}(z)=-\alpha\left(X_{H}(z), X_{H}(z)\right) .
$$

(For the specific case of Lie Poisson systems, this is equation (6.12) with $h=k$ ). Notice that the relative equilibrium is isolated in the leaf (coadjoint orbit), which follows from our nondegeneracy assumption. Thus, we see that in the leaf (coadjoint 
orbit), $\dot{H}$ is strictly negative in a deleted neighborhood of the equilibrium. The Liapunov instability now follows from Liapunov's instability theorem (see Theorem III, page 38 of LaSalle and Lefschetz [1963]) and thus we get the first part of the theorem. We get the second part of the theorem by applying Proposition 4.1 of [BKMR] (which is based on Hahn [1967]) using the Liapunov function $W=\delta^{2} H\left(z_{e}\right)$.

In $[\mathrm{BKMR}]$ it was necessary to modify the energy function to a new function called the Chetaev function, as in some of the original work of Chetaev (who treated the special case of Abelian groups). We observe that in the above theorem, we do not need to modify the Hamiltonian to the Chetaev function; that is, $\dot{H}$ is already positive definite, being (in the dual of the Lie algebra case) the square norm of the gradient of the Hamiltonian relative to the normal metric. However, when we do couple the Lie algebra case to that of internal variables below, it will indeed be necessary to modify the Hamiltonian to a Chetaev like functional.

We remark that the preceding theorem admits a slight generalization that could be of interest. Namely, if in the dissipative term one replaces $H$ by $K$, and if $H$ and $K$ Poisson commute and have the same critical point (the equilibrium), then if one replaces the hypotheses on $H$ in the theorem by the corresponding ones on $K$, it remains valid by the same proof applied to the Liapunov function $K$.

\section{Lie-Poisson Examples}

\subsection{The Rigid Body and the Landau-Lifschitz equations}

The calculations needed to show that the general theory applied to the dual of the Lie algebra of the rotation group gives the dissipative terms given in $\S 2$ are straightforward following the outline given. We can omit the details.

\subsection{Ideal Fluids}

We now give the calculations for the results stated in $\S 2$.

For incompressible fluids moving in a region $\Omega$ of $\mathbb{R}^{d}$, or, more generally a smooth oriented Riemannian manifold, the phase space is $\mathfrak{X}_{\text {div }}(\Omega)^{*}$ which we identify with $\mathfrak{X}_{\text {div }}(\Omega)$, the Lie algebra of vector fields that are divergence free and parallel to the boundary by the $L^{2}$-inner product. The $(+)$ Lie-Poisson bracket is

$$
\{F, H\}(v)=-\int_{\Omega} g\left(v,\left[\frac{\delta F}{\delta v}, \frac{\delta H}{\delta v}\right]\right) d x,
$$

where $g$ is the Riemannian metric on $\Omega$ and $d x$ is the associated volume element. There is a minus in front of the integral sign because the Jacobi-Lie bracket of vector fields is the right Lie algebra bracket for the group of volume preserving diffeomorphisms on $\Omega$. In general, Hamilton's equations for the $(+)$ Lie-Poisson structure are

$$
\frac{d \mu}{d t}=-\operatorname{ad}_{\delta H / \delta \mu}^{*} \mu
$$


We compute the ad*action in our case. Let $u, v, w \in \mathfrak{X}_{\text {div }}(\Omega)$. Then

$$
\begin{aligned}
\left\langle-\operatorname{ad}_{u}^{*} v, w\right\rangle & =-\langle v,[u, w]\rangle \\
& =-\int_{\Omega} g(v,[u, w]) d x \\
& =-\int_{\Omega} v^{b} \cdot\left(£_{u} w\right) d x
\end{aligned}
$$

where ${ }^{b}$ denotes the index lowering action defined by the metric $g$ on $\Omega$ and where $£$ denotes the Lie derivative. However,

$$
£_{u}\left(v^{b} \cdot w d x\right)=\left(£_{u} v^{b}\right) \cdot w d x+v^{b} \cdot\left(£_{u} w\right) d x+\left(v^{b} \cdot w\right) £_{u}(d x) .
$$

The last term vanishes since $u \in \mathfrak{X}_{\operatorname{div}}(\Omega)$. Thus the above relation becomes:

$$
\left\langle-\operatorname{ad}_{u}^{*} v, w\right\rangle=\int_{\Omega}\left(£_{u} v^{b}\right) \cdot w d x-\int_{\Omega} £_{u}\left(v^{b} \cdot w d x\right) .
$$

The second integral vanishes:

$$
\int_{\Omega} £_{u}\left(v^{b} \cdot w d x\right)=\int_{\partial \Omega} \mathbf{i}_{u}\left(v^{b} \cdot w d x\right)=\int_{\partial \Omega}\left(v^{b} \cdot w\right)(u \cdot \mathbf{n}) d a=0,
$$

where $\mathbf{n}$ is the outward unit normal to $\partial \Omega$ and $d a$ is the induced volume on the boundary. Denoting by $\mathbf{P}: \mathfrak{X}(\Omega) \rightarrow \mathfrak{X}_{\text {div }}(\Omega)$ the Hodge projection and by ${ }^{\sharp}$ the index raising action defined by the Riemannian metric $g$, we get

$$
\begin{aligned}
\left\langle-\operatorname{ad}_{u}^{*} v, w\right\rangle & =\int_{\Omega}\left(£_{u} v^{b}\right) \cdot w d x=\int_{\Omega} g\left(\left(£_{u} v^{b}\right)^{\sharp}, w\right) d x \\
& =\int_{\Omega} g\left(\mathbf{P}\left(\left(£_{u} v^{b}\right)^{\sharp}\right), w\right) d x=\left\langle\mathbf{P}\left(\left(£_{u} v^{b}\right)^{\sharp}\right), w\right\rangle,
\end{aligned}
$$

whence

$$
-\operatorname{ad}_{u}^{*} v=\mathbf{P}\left(\left(£_{u} v^{b}\right)^{\sharp}\right) .
$$

Consequently, denoting by $\Lambda: T^{*} \mathfrak{X}_{\text {div }}(\Omega) \rightarrow T \mathfrak{X}_{\text {div }}(\Omega)$ the Poisson structure defined by

$$
\begin{aligned}
\left\langle\mathbf{d} H(v), \Lambda_{v}(\mathbf{d} F(v))\right\rangle & =\{F, H\}(v) \\
& =\left\langle\operatorname{ad}_{\frac{\delta F}{\delta v}}^{*} v, \frac{\delta H}{\delta v}\right\rangle \\
& =\left\langle-\mathbf{P}\left(\left(£_{\frac{\delta F}{\delta v}} v^{b}\right)^{\sharp}\right), \frac{\delta H}{\delta v}\right\rangle,
\end{aligned}
$$

we get

$$
\Lambda_{v}(u)=-\mathbf{P}\left(\left(£_{u} v^{b}\right)^{\sharp}\right) .
$$

For example, if we choose $\Gamma=$ identity and $C(v)=1 / \alpha$ for $\alpha$ a strictly positive constant, the dissipative forcing term has the expression

$$
\begin{aligned}
\alpha \operatorname{ad}_{\Gamma\left(\operatorname{ad}_{\delta H / \delta v}^{*} v\right)}^{*} v & =-\alpha \mathbf{P}\left(\left(£_{\left(\operatorname{ad}_{v}^{*} v^{b}\right)^{\sharp}} v^{b}\right)^{\sharp}\right) \\
& =\alpha \mathbf{P}\left(\left(£_{u(v)} v^{b}\right)^{\sharp}\right),
\end{aligned}
$$


where $u(v)=\mathbf{P}\left(\left(£_{v} v^{b}\right)^{\sharp}\right)$.

It is instructive to verify directly that $d H / d t<0$ on the solutions of the dissipative system

$$
\frac{\partial v}{\partial t}+\nabla_{v} v=-\nabla p+\alpha \mathbf{P}\left(\left(£_{u(v)} v^{b}\right)^{\sharp}\right) .
$$

Recall the formula

$$
£_{v}\left(v^{b}\right)=\left(\nabla_{v} v\right)^{b}+\frac{1}{2} \mathbf{d}\|v\|^{2} .
$$

Therefore the equation above becomes

$$
\frac{\partial v^{b}}{\partial t}+£_{v}\left(v^{b}\right)=-\mathbf{d}\left(p+\frac{1}{2}\|v\|^{2}\right)+\alpha\left[\mathbf{P}\left(\left(£_{u(v)} v^{b}\right)^{\sharp}\right)\right]^{b}
$$

and so we get:

$$
\begin{aligned}
\frac{d H}{d t} & =\frac{d}{d t} \frac{1}{2} \int_{\Omega}\|v\|^{2} d x=\frac{d}{d t} \frac{1}{2} \int_{\Omega} v^{b} \cdot v d x \\
& =\int_{\Omega} \frac{d v^{b}}{d t} \cdot v d x=\alpha \int_{\Omega}\left[\mathbf{P}\left(\left(£_{u(v)} v^{b}\right)^{\sharp}\right)\right]^{b} \cdot v d x \\
& =\alpha \int_{\Omega} £_{u(v)}\left(v^{b}\right) \cdot v d x=-\alpha \int_{\Omega} v^{b} \cdot £_{u(v)} v d x \\
& =\alpha \int_{\Omega} v^{b} \cdot £_{v} u(v) d x=-\alpha \int_{\Omega} £_{v}\left(v^{b}\right) \cdot u(v) d x \\
& =-\alpha \int_{\Omega}\left\|\mathbf{P}\left(\left(£_{u(v)} v^{b}\right)^{\sharp}\right)\right\|^{2} d x<0 .
\end{aligned}
$$

The vorticity form of the equations, as stated in the introduction is readily verified by taking the differential of the dissipative equations for $v^{b}$ and recalling that $\omega=\mathbf{d} v^{b}$.

\subsection{The Vlasov-Poisson Equations}

The equations of motion for a one species collisionless plasma moving in a background static ion field in $\mathbb{R}^{n}$ are given by the Vlasov-Poisson equations

$$
\frac{d f}{d t}+v \cdot \frac{\partial f}{\partial x}-\frac{q}{m} \frac{\partial \phi_{f}}{\partial x} \cdot \frac{\partial f}{\partial v}=0, \quad \nabla^{2} \phi_{f}(x)=-\rho_{f}(x)=q\left(\int f(x, v) d v-1\right),
$$

where $\partial / \partial x, \partial / \partial v$ denote the gradients with respect to $x$ and $v$ respectively, $\nabla^{2}$ is the Laplacian in the $x$-variable, $f(x, v)$ is the phase space density satisfying

$$
\iint f(x, v) d x d v=1
$$

$q$ is the charge, $m$ is the mass, and $\rho_{f}(x)$ is the total charge density of the plasma. We assume that $f$ is either periodic in $x$ or has appropriate asymptotic behavior as $x$ tends to infinity and that $f$ decays for $v$ approaching infinity. 
For two functions $g(x, v), h(x, v)$ define

$$
\{g, h\}=\frac{1}{m}\left(\frac{\partial g}{\partial x} \cdot \frac{\partial h}{\partial v}-\frac{\partial h}{\partial x} \cdot \frac{\partial g}{\partial v}\right)
$$

the canonical Poisson bracket in $(x, v)$-space. Under the above hypotheses on the functions considered, it can be shown by integration by parts that the $L^{2}$-inner product is invariant on the Lie algebra $\mathfrak{g}$ of functions of $(x, v)$ endowed with the above Poisson bracket.

The Vlasov-Poisson equations can be equivalently written in the form

$$
\frac{\partial f}{\partial t}=\left\{\mathcal{H}_{f}, f\right\}
$$

where

$$
\mathcal{H}_{f}=\frac{1}{2} m^{2}\|v\|^{2}+q \phi_{f}(x)
$$

is the one particle Hamiltonian. The total energy of the system has the expression

$$
H(f)=\frac{1}{2} \int m\|v\|^{2} f(x, v) d x d v+\frac{1}{2} \int q \phi_{f}(x) \rho_{f}(x) d x .
$$

and one has $\delta H / \delta f=\mathcal{H}_{f}$.

The Vlasov-Poisson equations are Hamiltonian on the dual of the Lie algebra $\mathfrak{g}$ of functions of $(x, v)$ under the canonical Poisson bracket. We identify $\mathfrak{g}$ with its dual by identifying functions with densities using the Liouville volume element, denoted by $d x d v$ (see Morrison [1980,1982] and Marsden and Weinstein [1982]). The (+) Lie-Poisson bracket has the expression

$$
\{F, K\}_{\mathrm{LP}}=\int f\left\{\frac{\delta F}{\delta f}, \frac{\delta K}{\delta f}\right\} d x d v .
$$

The Hamiltonian vector field of a functional $F$ evaluated at a plasma density function $f \in \mathfrak{g}^{*}$ is given by

$$
X_{F}(f)=\left\{\frac{\delta F}{\delta f}, f\right\} d x d v
$$

Since $\delta H / \delta f=\mathcal{H}_{f}$, the Vlasov-Poisson equations are equivalent to $\dot{F}=\{F, H\}_{\mathrm{LP}}$ for $H$, the total energy of the plasma.

The equations with dissipation have the usual form

$$
\dot{F}=\{F, H\}_{\mathrm{LP}}-\{\{F, H\}\},
$$

where the symmetric bracket is given by

$$
\{\{F, K\}\}=\alpha \int\left\langle X_{F}, X_{K}\right\rangle d x d v .
$$


Due to the invariance of the $L^{2}$-inner product, $\Gamma$ is the identity in this case. Thus the symmetric bracket is given by

$$
\begin{aligned}
\{\{F, K\}\} & =\int_{P}\left\{\frac{\delta F}{\delta f}, f\right\} \cdot\left\{\frac{\delta K}{\delta f}, f\right\} d x d v \\
& =\int_{P} \frac{\delta F}{\delta f} \cdot\left\{f,\left\{\frac{\delta K}{\delta f}, f\right\}\right\} d x d v
\end{aligned}
$$

and hence the Vlasov-Poisson equation with dissipation is

$$
\dot{f}+\left\{f, \mathcal{H}_{f}\right\}=\alpha\left\{f,\left\{f, \mathcal{H}_{f}\right\}\right\}
$$

where $\mathcal{H}_{f}$ is the one particle Hamiltonian and $\alpha$ is a strictly positive constant. Since the equations of stellar dynamics are identical in form to this system (with attractive gravitational rather than repulsive electrical forces), the same formalism applies to them as well. See Kandrup [1991] and Kandrup and Morrison [1992].

\subsection{The Heavy Top}

It is known from Lewis, Ratiu, Simo and Marsden [1992] that there are equilibria for the heavy top with a fixed point that exhibit gyroscopic stabilization, and these equilibria are thus interesting from the point of view of dissipation induced instabilities. We recall that the equations are of Lie-Poisson form on the dual of the Lie algebra of the Euclidean group of $\mathbb{R}^{3}$. They are given by

$$
\begin{aligned}
& \dot{\Pi}=\Pi \times \Omega+g \gamma \times M, \\
& \dot{\gamma}=\gamma \times \Omega,
\end{aligned}
$$

where $\Pi=\mathbb{I} \Omega, \mathbb{I}$ is the moment of inertia tensor, $M$ is the constant center of mass vector, $\gamma$ is the direction of gravity as seen from the body and $g$ is the acceleration due to gravity. The Hamiltonian is

$$
H=\frac{1}{2} \Pi \cdot \Omega+g \gamma \cdot M
$$

and the Lie-Poisson bracket is

$$
\{F, K\}(\Pi, \gamma)=-(\Pi, \gamma) \cdot\left(\nabla_{\Pi} F \times \nabla_{\Pi} K, \nabla_{\Pi} F \times \nabla_{\gamma} K+\nabla_{\gamma} F \times \nabla_{\Pi} K\right) .
$$

Computing the double bracket from the general theory above, with $\Gamma$ being the identity, one finds that the dissipative equations are:

$$
\begin{aligned}
\dot{\Pi} & =\Pi \times \Omega+g \gamma \times M+\alpha[\Pi \times(\Pi \times \Omega+g \gamma \times M)+\gamma \times(\gamma \times \Omega)], \\
\dot{\gamma} & =\gamma \times \Omega+\alpha[\gamma \times(\Pi \times \Omega+g \gamma \times M)] .
\end{aligned}
$$

This form of the dissipation automatically preserves the coadjoint orbits; that is, it preserves the length of $\gamma$ and the orthogonality of $\gamma$ and $\Pi$. Thus, this dissipation will 
have the property that when it is added to the equations, it will preserve relative equilibria and any equilibrium that is energetically a saddle point but which has eigenvalues on the imaginary axis will become spectrally (and hence linearly and nonlinearly) unstable when the dissipation is added; equilibria with this property are exhibited in Lewis, Ratiu, Simo and Marsden [1992].

\section{Instability for Systems with both Internal and Double Bracket Dissipation}

In [BKMR], we considered mechanical systems on configuration spaces $Q$ that are invariant under the action of a group $G$ on $Q$. As before, the Lie algebra of $G$ will be denoted $\mathfrak{g}$. In this context, the variables in the problem divide into group (sometimes called rigid) variables and into internal variables. We considered the effect of adding dissipation to the internal variables and showed that if the second variation of the augmented energy is indefinite, and if the rigid-internal coupling matrix $C$ satisfies a nondegeneracy condition (namely that $C$ be surjective as a map from the internal space to the rigid space, i.e., that its transpose $C^{T}$ is injective), then the addition of this internal dissipation induced a spectral instability in the equations linearized at a relative equilibrium. Here we show that there is a similar theorem for the case of the addition of double bracket Lie-Poisson dissipation of the sort considered in this paper. We also allow a combination of internal and Lie-Poisson dissipation. Interestingly, the details of the argument in the present case are different than those in the purely internal dissipative case, and so we will give the proof.

We will need to recall the form of the linearized equations at a relative equilibrium with internal dissipation. By making use of the block diagonalization theory of Simo, Lewis and Marsden [1991], they are shown in [BKMR] to be the following:

$$
\left.\begin{array}{rl}
\dot{r} & =-L_{\mu}^{-1} A_{\mu} r-L_{\mu}^{-1} C M^{-1} p \\
\dot{q} & =M^{-1} p \\
\dot{p} & =-C^{T} L_{\mu}^{-1} A_{\mu} r-\Lambda q-\tilde{S} M^{-1} p-R M^{-1} p .
\end{array}\right\}
$$

Here, the variable $r$ is a dynamic variable in the linear space $\mathcal{V}_{\mathrm{RIG}}$, which is isomorphic to the tangent space to the coadjoint orbit $\operatorname{Orb}_{\mu} \subset \mathfrak{g}^{*}$ that passes through the value $\mu$ of the momentum of the relative equilibrium in question. The operator $L_{\mu}$ is the Kirillov-Kostant-Souriau symplectic operator on the coadjoint orbit evaluated at $\mu$, so that it is skew symmetric. Thus, its inverse is the Poisson tensor. The symmetric operator $A_{\mu}$ is the linearized energy operator for the rigid variables. The operator $C$ is the coupling matrix, coupling the internal variables and the rigid variables, and $M$ is the positive definite symmetric mass matrix. The variables $q$ and $p$ are the (linearized) internal configuration and momentum variables. The matrix $\Lambda$ is the linearized internal amended potential energy (so it includes the centrifugal energy), $\tilde{S}$ is a skew symmetric gyroscopic term and $R$ is the symmetric Rayleigh dissipation matrix for the internal variables. See [BKMR] for the explicit expression 
for these equations. In that paper, we assumed full dissipation in the sense that the matrix $R$ was assumed to be positive definite and that the coupling matrix $C$ was surjective; in this section, we assume only that the matrix $R$ is positive semidefinite. In fact, provided a condition spelled out below is satisfied, the matrix $R$ can be allowed to be zero. In the case that $R$ is zero, the condition reduces to the condition that the matrix $C$ is injective (rather than surjective as before). Thus, we allow partial internal dissipation in this theorem. We modify the above linearized equations and consider the system

$$
\left.\begin{array}{rl}
\dot{r} & =-L_{\mu}^{-1} A_{\mu} r-L_{\mu}^{-1} C M^{-1} p-G^{-1} A_{\mu} r \\
\dot{q} & =M^{-1} p \\
\dot{p} & =-C^{T} L_{\mu}^{-1} A_{\mu} r-\Lambda q-\tilde{S} M^{-1} p-R M^{-1} p .
\end{array}\right\}
$$

Here, the matrix $G$ will be assumed to be symmetric and positive definite. Note that this extra term is dissipation of the form that we considered earlier where $G$ represents the normal metric on the coadjoint orbit. With the dissipative terms $R$ and $G$ omitted, these equations are Hamiltonian with the Hamiltonian function given by the second variation of the augmented Hamiltonian $\delta^{2} H_{\xi}$ (where $\xi$ is the Lie algebra element defining the underlying relative equilibrium); this second variation is the quadratic form associated to the block diagonal matrix

$$
\left[\begin{array}{ccc}
A_{\mu} & 0 & 0 \\
0 & \Lambda & 0 \\
0 & 0 & M^{-1}
\end{array}\right]
$$

One can check directly that the following dissipation equation holds:

$$
\frac{d \delta^{2} H_{\xi}}{d t}=-\left(M^{-1} p\right)^{T} R\left(M^{-1} p\right)-\left(A_{\mu} r\right)^{T} G^{-1}\left(A_{\mu} r\right)
$$

Of course, because the right hand side is only semidefinite in the variables $(r, q, p)$, one cannot directly use the energy equation alone to conclude instability. This is a central difficulty that was addressed in the work of Chetaev and generalized in $[\mathrm{BKMR}]$. We consider the following nondegeneracy hypothesis:

(D) If $v$ is a vector in the internal space such that $C v=0$ and $R v=0$, then $v=0$.

Note that this hypothesis is equivalent to saying that the matrix $C^{T} C+R$ is positive definite.

Theorem 9.1 Assume that $G$ is symmetric and positive definite, and that either $A_{\mu}$ or $\Lambda$ has at least one negative eigenvalue. Also assume that $R$ is positive semidefinite and condition (D) holds. Then the system (9.2) is Liapunov unstable. If, in addition, the dissipation added is sufficiently small, then the equilibrium is spectrally unstable as well (i.e., it has some eigenvalues in the right half plane). Thus, if the 
dissipation of a given nonlinear system is such that the linearized equations at a relative equilibrium have the form (9.1), and the dissipation is sufficiently small, then the relative equilibrium is nonlinearly unstable.

Proof We will be writing various matrices using block form; when doing so, we will write them consistently in the order $(r, q, p)$. We consider the Chetaev-type function defined as follows:

$$
W(r, q, p)=\frac{1}{2} p \cdot M^{-1} p+\frac{1}{2} q \cdot \Lambda q+\frac{1}{2} r \cdot A_{\mu} r+\beta B q \cdot M^{-1} p+\alpha D r \cdot M^{-1} p .
$$

A priori, the matrix $\Lambda$ is not required to be invertible, but the same remarks as in $[\mathrm{BKMR}]$ (see the proof of Theorem 3.1 of that paper) allow one to reduce to the case in which $\Lambda$ is nonsingular, so we will make this assumption. We choose a positive definite matrix $K$ on the internal configuration variables (the freedom to choose $K$ is important only to deal with the possibility that $\Lambda$ is degenerate; if $\Lambda$ is nondegenerate, one can take $K$ to be the identity) and let $D=C^{T} K L_{\mu}$ and $B=M K^{-1} \Lambda$. Note that the choice of $D$ here is not the same as in the case of purely internal dissipation; in that case, we chose $\alpha=\beta$ and had a third term in the definition of $W$-this will not be the case here. We choose $\beta=\alpha^{3 / 2}$ and choose $\alpha$ to be sufficiently small. As in [BKMR], a straightforward but somewhat lengthy computation shows that the time derivative of $-W$ is given in block partitioned form by

$$
-\dot{W}=\left[\begin{array}{ccc}
A_{11} & A_{12} & A_{13} \\
A_{12}^{T} & A_{22} & A_{23} \\
A_{13}^{T} & A_{23}^{T} & A_{33}
\end{array}\right],
$$

where the matrices in this array are given by

$$
\begin{aligned}
A_{11}= & \frac{\alpha}{2}\left(D^{T} M^{-1} C^{T} L_{\mu}^{-1} A_{\mu}-A_{\mu} L_{\mu}^{-1} C M^{-1} D\right)+A_{\mu} G^{-1} A_{\mu}, \\
A_{22}= & \frac{\beta}{2}\left(B^{T} M^{-1} \Lambda+\Lambda M^{-1} B\right), \\
A_{33}= & M^{-1} R M^{-1}-\frac{\beta}{2}\left(M^{-1} B^{T} M^{-1}+M^{-1} B M^{-1}\right) \\
& +\frac{\alpha}{2}\left(-M^{-1} C^{T} L_{\mu}^{-1} D^{T} M^{-1}+\left(M^{T}\right)^{-1} D L_{\mu}^{-1} C M^{-1}\right), \\
A_{12}= & \frac{\alpha}{2} D^{T} M^{-1} \Lambda-\frac{\beta}{2} A_{\mu} L_{\mu}^{-1} C M^{-1} B, \\
A_{13}= & \frac{\alpha}{2}\left(D^{T} M^{-1}(R+\tilde{S}) M^{-1}-A_{\mu}\left(L_{\mu}^{-1}+G^{-1}\right) D^{T} M^{-1}\right), \\
A_{23}= & \frac{\beta}{2} B^{T} M^{-1}(R+\tilde{S}) M^{-1}
\end{aligned}
$$

We now show that $-\dot{W}$ is positive definite for $\alpha$ sufficiently small. To do this, it is sufficient to show that the matrices $A_{11}, \tilde{A}_{22}=A_{22}-A_{12}^{T} A_{11}^{T} A_{12}$ and

$$
\begin{aligned}
\tilde{A}_{33}= & A_{33}-A_{13}^{T} A_{11}^{-1} A_{13} \\
& -\left(A_{23}^{T}-A_{13} A_{11}^{-1} A_{12}\right)\left(A_{22}-A_{12}^{T} A_{11}^{-1} A_{12}\right)^{-1}\left(A_{23}-A_{12}^{T} A_{11}^{-1} A_{13}\right)(9.13)
\end{aligned}
$$


are positive definite. This is proved in [BKMR]; see Lemma 2.11 and equation (3.15). However, by direct inspection of the forms of these matrices, one finds that

$$
\begin{aligned}
A_{11} & =A_{\mu} G^{-1} A_{\mu}+O(\alpha), \\
\tilde{A}_{22} & =(\alpha)^{3 / 2} \Lambda K^{-1} \Lambda+O\left(\alpha^{2}\right), \\
\tilde{A}_{33} & =\alpha M^{-1}\left(C^{T} K C+R\right) M^{-1}+O\left((\alpha)^{3 / 2}\right) .
\end{aligned}
$$

Thus, under the given condition (D), these matrices are all positive definite if $\alpha$ is small enough. Clearly $W$ itself is indefinite if $\alpha$ is small enough, and so by Liapunov's instability theorem (see Lemma 3.2 of [BKMR]) we get the first part of the theorem. We get the second part of the theorem by Proposition 4.1 of the same paper.

One can ask in this context, what form of dissipation should be added to the original nonlinear system so that its linearization at a relative equilibrium will have the stated form. We believe that the answer to this is that the force function should be divided into a vertical and a horizontal part and that the vertical part should be of double bracket form and that the horizontal part should be of Rayleigh dissipation type. Here, the horizontal and vertical decomposition should be done relative to a connection as in the reduced Euler-Lagrange equations in Marsden and Scheurle [1993b]. We plan to investigate the global aspects of such splittings in another publication, but we will see how this works in the specific example of the rigid body with internal rotors below.

\section{The Rigid body with rotors}

Here we illustrate Theorem 9.1 using a rigid body with two or three symmetric internal rotors. In the case of two rotors, we will require no internal dissipation, i.e., we can choose $R=0$. As we will see, if there are three internal rotors, then the rotor about the axis of rotation must have its own internal dissipation for hypothesis (D) to hold.

We first consider the case of three rotors subject to internal friction with the overall rotation subject to double bracket dissipation. We will shortly specialize to the case of two rotors with no internal dissipation. A steady spin about the minor axis of the locked inertia tensor ellipsoid (i.e., the long axis of the body), is a relative equilibrium. Without friction, this system can experience gyroscopic stabilization and the second variation of the augmented Hamiltonian can be indefinite. We will show that this is an unstable relative equilibrium with double bracket dissipation added.

The full equations of motion with both internal and double bracket dissipation are (see Krishnaprasad [1985] and Bloch, Krishnaprasad, Marsden, and Sanchez de 
Alvarez [1992]):

$$
\begin{aligned}
& \left(\mathbb{I}_{\text {lock }}-\mathbb{I}_{\text {rotor }}\right) \dot{\Omega}=\left(\mathbb{I}_{\text {lock }} \Omega+\mathbb{I}_{\text {rotor }} \Omega_{r}\right) \times \Omega \\
& +\alpha\left(\mathbb{I}_{\text {lock }} \Omega+\mathbb{I}_{\text {rotor }} \Omega_{r}\right) \times\left(\left(\mathbb{I}_{\text {lock }} \Omega+\mathbb{I}_{\text {rotor }} \Omega_{r}\right) \times \Omega\right), \\
& \dot{\Omega}_{r}=-\left(\mathbb{I}_{\text {lock }}-\mathbb{I}_{\text {rotor }}\right)^{-1}\left(\mathbb{I}_{\text {lock }} \Omega+\mathbb{I}_{\text {rotor }} \Omega_{r}\right) \times \Omega-R \Omega_{r}, \\
& \dot{A}=A \hat{\Omega}, \\
& \dot{\theta}_{r}=\Omega_{r} .
\end{aligned}
$$

Here, $\alpha$ is a positive constant, $Q=S O(3) \times S^{1} \times S^{1}$ (three factors if there are three rotors) and $G=S O(3)$. Also $A \in S O(3)$ denotes the attitude/orientation of the carrier rigid body relative to an inertial frame, $\Omega \in \mathbb{R}^{3}$ is the body angular velocity of the carrier, $\Omega_{r} \in \mathbb{R}^{3}$ is the vector of angular velocities of the rotors in the body frame (with third component set equal to zero) and $\theta_{r}$ is the ordered set of rotor angles in body frame (again, with third component set equal to zero). Further, $\mathbb{I}_{\text {lock }}$ denotes the moment of inertia of the body and locked rotors in the body frame and $\mathbb{I}_{\text {rotor }}$ is the $3 \times 3$ diagonal matrix of rotor inertias. Finally, $R=\operatorname{diag}\left(R_{1}, R_{2}, R_{3}\right)$ is the matrix of rotor dissipation coefficients, $R_{i} \geq 0$.

In Hamiltonian form, these equations read:

$$
\begin{aligned}
\dot{\Pi} & =\Pi \times \Omega+\alpha \Pi \times(\Pi \times \Omega), \\
\dot{\ell} & =-\mathbb{I}_{\text {rotor }} R \Omega_{r},
\end{aligned}
$$

where $\Pi=\mathbb{I}_{\text {lock }} \Omega+\mathbb{I}_{\text {rotor }} \Omega_{r}$ and $\ell=\mathbb{I}_{\text {rotor }}\left(\Omega+\Omega_{r}\right)$. Here, $\Omega=\mathbb{J}^{-1}(\Pi-\ell)$, where $\mathbb{J}=\mathbb{I}_{\text {lock }}-\mathbb{I}_{\text {rotor }}$. The Hamiltonian is

$$
H=\frac{1}{2}\left\langle\mathbb{J}^{-1}(\Pi-\ell), \Pi-\ell\right\rangle+\frac{1}{2}\left\langle\mathbb{I}_{\text {rotor }}^{-1} \ell, \ell\right\rangle .
$$

Notice by direct calculation that

$$
\frac{d}{d t}\|\Pi\|^{2}=0
$$

and that

$$
\frac{d}{d t} H=-\alpha\|\Pi \times \Omega\|^{2}-\left\langle R \Omega_{r}, \Omega_{r}\right\rangle .
$$

We let

$$
\left.\begin{array}{rl}
\mathbb{I}_{\text {lock }} & =\operatorname{diag}\left(B_{1}, B_{2}, B_{3}\right), \\
\mathbb{I}_{\text {rotor }} & =\operatorname{diag}\left(J_{1}^{1}, J_{2}^{2}, J_{3}^{3}\right), \\
\mathbb{I}_{\text {lock }}-\mathbb{I}_{\text {rotor }} & =\operatorname{diag}\left(A_{1}, A_{2}, A_{3}\right) .
\end{array}\right\}
$$


Assume that $B_{1}>B_{2}>B_{3}$.

Now we specialize to the case of two rotors and no internal dissipation. We set $R_{i}=0$ and $J_{3}^{3}=0$. Consider the relative equilibrium for (10.1) defined by, $\Omega^{e}=(0,0, \omega)^{T} ; \Omega_{r}^{e}=(0,0,0)^{T}$ and $\theta_{r}=\theta_{r}^{e}$ an arbitrary constant. This corresponds to a steady minor axis spin of the rigid body with the two rotors non-spinning. Linearization about this equilibrium yields,

$$
\begin{aligned}
\left(\mathbb{I}_{\text {lock }}-\right. & \left.\mathbb{I}_{\text {rotor }}\right) \delta \dot{\Omega}=\left(\mathbb{I}_{\text {lock }} \delta \Omega+\mathbb{I}_{\text {rotor }} \delta \Omega_{r}\right) \times \Omega^{e}+\left(\mathbb{I}_{\text {lock }} \Omega^{e}\right) \times \delta \Omega \\
& +\alpha\left(\mathbb{I}_{\text {lock }} \Omega^{e}\right) \times\left(\left(\mathbb{I}_{\text {lock }} \Omega^{e}\right) \times \delta \Omega\right)+\alpha\left(\mathbb{I}_{\text {lock }} \Omega^{e}\right) \times\left(\left(\mathbb{I}_{\text {lock }} \delta \Omega+\mathbb{I}_{\text {rotor }} \delta \Omega_{r}\right) \times \Omega^{e}\right) \\
\delta \dot{\Omega}_{r}= & -\left(\mathbb{I}_{\text {lock }}-\mathbb{I}_{\text {rotor }}\right)^{-1}\left[\left(\mathbb{I}_{\text {lock }} \delta \Omega+\mathbb{I}_{\text {rotor }} \delta \Omega_{r}\right) \times \Omega^{e},\right. \\
& \left.+\left(\mathbb{I}_{\text {lock }} \Omega^{e}\right) \times \delta \Omega\right]-R \delta \Omega_{r}, \\
\delta \dot{\theta}_{r}= & \delta \Omega_{r} .
\end{aligned}
$$

It is easy to verify that $\delta \dot{\Omega}_{3}=0$, which reflects the choice of relative equilibrium. Similarly $\delta \dot{\Omega}_{r_{3}}=0$. We will now apply Theorem 8.1 in the case of $\Lambda=0$.

Dropping the kinematic equations for $\delta \theta_{r}$ we have the "reduced" linearized equations

$$
\left[\begin{array}{c}
\delta \dot{\Omega}_{r_{1}} \\
\delta \dot{\Omega}_{r_{2}} \\
\delta \dot{\Omega}_{1} \\
\delta \dot{\Omega}_{2}
\end{array}\right]=\left[\begin{array}{cccc}
0 & \frac{-J_{2}^{2} \omega}{A_{1}} & 0 & \frac{B_{3}-B_{2}}{A_{1}} \omega \\
\frac{J_{1}^{1} \omega}{A_{2}} & 0 & \frac{B_{1}-B_{3}}{A_{2}} \omega & 0 \\
0 & \frac{J_{2}^{2} \omega}{A_{1}} & \frac{B_{3} \omega^{2}\left(B_{3}-B_{1}\right)}{A_{2}} & \frac{B_{2}-B_{3}}{A_{1}} \omega \\
\frac{-J_{1}^{1} \omega}{A_{2}} & 0 & \frac{B_{3}-B_{1}}{A_{2}} \omega & \frac{B_{3} \omega^{2}\left(B_{2}-B_{3}\right)}{A_{2}}
\end{array}\right]\left[\begin{array}{c}
\delta \Omega_{r_{1}} \\
\delta \Omega_{r_{2}} \\
\delta \Omega_{1} \\
\delta \Omega_{2}
\end{array}\right]
$$

Assume that $\omega \neq 0$ (nondegeneracy of the relative equilibrium). Then the above equations are easily verified to be in the normal form (9.2) with $R=0$, upon making 
the identifications, $p=\left(\delta \Omega_{r_{1}}, \delta \Omega_{r_{2}}\right), q=\left(\delta \theta_{r_{1}}, \delta \theta_{r_{2}}\right), r=\left(\delta \Omega_{1}, \delta \Omega_{2}\right)$, and

$$
\begin{aligned}
L_{\mu} & =\left(\begin{array}{cc}
0 & -1 / \omega \\
1 / \omega & 0
\end{array}\right) ; C=\left(\begin{array}{cc}
-1 & 0 \\
0 & -1
\end{array}\right) ; \tilde{S}=\left(\begin{array}{cc}
0 & \omega \\
-\omega & 0
\end{array}\right) ; \\
A_{\mu} & =\left(\begin{array}{cc}
\frac{B_{3}-B_{1}}{A_{2}} & 0 \\
0 & \frac{B_{3}-B_{2}}{A_{1}}
\end{array}\right) ; M^{-1}=\left(\begin{array}{cc}
\frac{J_{1}^{1}}{A_{2}} & 0 \\
0 & \frac{J_{2}^{2}}{A_{1}}
\end{array}\right) ; \\
G^{-1} & =\left(\begin{array}{cc}
B_{3} & 0 \\
0 & B_{3}
\end{array}\right) .
\end{aligned}
$$

Since $B_{1}>B_{2}>B_{3}, A_{\mu}$ is negative definite. Also, $M$ and $G$ are positive definite, and $C$ is injective and thus all the hypotheses of Theorem 9.1 are satisfied. Thus the linearized system (10.4) or (10.5) displays dissipation-induced instability. That is, for $\alpha$ sufficiently small, the system will have at least one pair of eigenvalues in the right half plane.

For three rotors, the matrix $C$ will have three columns, with zeros in its last column and the first two columns as above; however, dissipation in the third rotor will reinstate the validity of hypothesis (D).

\section{Conclusions and Comments}

In this paper we have given a general method of constructing dissipative mechanisms that have the property that they preserve symplectic leaves of reduced spaces and dissipate energy. The most important case is that of the dual of a Lie algebra, in which case the dissipative term is shown to have a double bracket form considered by Brockett. We have shown that such dissipative terms induce specral (and hence linear and nonlinear) instabilities. For systems that come up in the energymomentum method, we have given a general dissipation induced instability theorem that couples the double bracket form of instability with internal dissipation, thereby complementing our previous results in $[\mathrm{BKMR}]$. We have shown that this theory applies to a number of interesting examples from ferromagnetics, ideal fluid flow and plasma dynamics.

Other systems beside Lagrangian and Hamiltonian systems also exhibit phenomena similar to dissipation induced instabilities. In particular, one gets these phenomena in reversible systems (see O'Reilley [1993]) and when one breaks the symmetry of a system (see Guckenheimer and Mahalov [1992] and Knobloch, Marsden and Mahalov [1994]).

In the future, we would like to analyse more infinite dimensional systems such as fluids and the Richardson number example of Abarbanel, Holm, Marsden and Ratiu [1986]. The Richardson number criterion for stability of shear flows in stratified fluids is especially interesting because one knows there that the ideal dissipationless

flow is energetically a saddle point yet is spectrally stable for Richardson number between 1/4 and 1. Another candidate would be a case like an ABC Euler flow 
on the sphere, as in Chern and Marsden [1990]. In the case of Euler flow, the techniques of Ebin and Marsden [1970] together with invariant manifold theory for infinite dimensional dynamical system should allow one to rigorously prove nonlinear instability from spectral instability.

Other examples that might be treated are damping mechanisms in planetary physics using the theory of rotating gravitational fluid masses of Riemann [1860], Poincaré $[1885,1892,1910]$, Chandrasekhar [1977], Lewis and Simo [1990], and Touma and Wisdom [1992]. We also expect that there will be a more detailed theory in the context of the semidirect product theory of Marsden, Ratiu and Weinstein [1984]. For example, one can treat the heavy top as either a Lie Poisson system or as a system with group $S^{1}$ and the rest of the variables internal variables. Comparison of the two methods would undoubtedly be of interest.

We note that the dissipation mechanism in the complex Ginzburg-Landau equations (thought of as a modification of the nonlinear Schrödinger equation) is of the type $\Lambda \alpha \Lambda \mathbf{d} K$ for a function $K$, where $K$ is a simple modification of the energy function. This dissipation, on the other hand does not preserve the momentum map associated with the phase shift symmetry or the translational symmetry. Using the methods of the present paper, such dissipation mechanisms can be constructed and these will presumably be interesting modifications of the nonlinear Schrödinger equation. We hope to investigate some of these issues in a forthcoming publication.

We also expect that one can develop an eigenvalue movement formula for the present context, as we did in [BKMR]. References relevant for this and other aspects of the general dissipation induced instability phenomenon include Thomson and Tait [1879], Poincaré [1885], Krein [1950], Ziegler [1956], Taussky [1961], Namachchivaya and Ariaratnam [1985], MacKay [1991], Haller [1992], and Pego and Weinstein [1992].

\section{Appendix. The Euler-Poincaré Equations for Gen- eral Lie Groups}

The main goal of this appendix is to prove Proposition 5.1 for general Lie groups. To accomplish this, we use a method of Alekseevski and Michor [1993] that constructs a large class of connections on a bundle of the form $G \times M$ with explicit formulae for the curvature.

We begin with their general construction. Assume that the Lie group $G$ acts on the left on a manifold $M$ and let $\alpha \in \Omega^{1}(M ; \mathfrak{g})$ be a given smooth $\mathfrak{g}$-valued one-form on $M$. For $u_{g} \in T_{g} G$ and $v_{m} \in T_{m} M$, define

$$
\Gamma^{l}(g, m)\left(u_{g}, v_{m}\right)=T_{g} R_{g^{-1}}\left(u_{g}\right)-\operatorname{Ad}_{g}\left(\alpha(m) \cdot v_{m}\right) .
$$

Then $\Gamma^{l} \in \Omega^{1}(G \times M ; \mathfrak{g})$. The left action of $G$ on $G \times M$ makes the projection $\operatorname{pr}_{2}: G \times M \rightarrow M$ into a principal left $G$-bundle and if $\xi \in \mathfrak{g}$, the infinitesimal generator it defines equals $\xi_{G \times M}(g, m)=\left(T_{e} R_{g}(\xi), 0\right)$. Therefore, by $(12.1)$ we see 
that $\Gamma^{l}\left(\xi_{G \times M}\right)=\xi$ and

$$
\begin{aligned}
\Gamma^{l}(h g, m)\left(T_{g} L_{h}\left(u_{g}\right), v_{m}\right) & =T_{h g} \cdot R_{(h g)^{-1}}\left(T_{g} L_{h}\left(u_{g}\right)\right)-\operatorname{Ad}_{h g}\left(\alpha(m) \cdot v_{m}\right) \\
& =\operatorname{Ad}_{h}\left(T_{g} R_{g^{-1}}\left(u_{g}\right)-\operatorname{Ad}_{g}\left(\alpha(m) \cdot v_{m}\right)\right) \\
& =\operatorname{Ad}_{h}\left(\Gamma^{l}(g, m)\left(u_{g}, v_{m}\right)\right)
\end{aligned}
$$

so that $\Gamma^{l}$ defines a left principal connection one-form on the trivial bundle $\mathrm{pr}_{2}$ : $G \times M \rightarrow M$. The horizontal subbundle $H \subset T(G \times M)$ is therefore given by

$$
H_{(g, m)}^{l}=\left\{\left(T_{e} L_{g}\left(\alpha(m) \cdot v_{m}\right), v_{m}\right) \mid v_{m} \in T_{m} M\right\} .
$$

To compute the curvature of this connection, we recall that if $\lambda, \rho \in \Omega^{1}(G ; \mathfrak{g})$ are defined by

$$
\lambda\left(u_{g}\right)=T_{g} L_{g^{-1}}\left(u_{g}\right), \quad \rho\left(u_{g}\right)=T_{g} R_{g^{-1}}\left(u_{g}\right),
$$

then the Maurer-Cartan structure equations state that

$$
\mathbf{d} \lambda+\frac{1}{2}\left[\lambda, \lambda \hat{]}=0, \quad \mathbf{d} \rho-\frac{1}{2}[\rho, \rho]=0,\right.
$$

where $\mathbf{d}$ is the exterior derivative and $[\cdot, \cdot]$ is the exterior product induced on $\mathfrak{g}$ by its Lie algebra bracket. Our coefficient conventions for $[\cdot, \cdot]$ are the following: if $\alpha, \beta \in \Omega^{1}(M ; \mathfrak{g})$ then

$$
[\alpha, \beta] \hat{(u, v)}=[\alpha(u), \beta(v)]-[\alpha(v), \beta(u)]=[\beta, \alpha \hat{]}(u, v) .
$$

Finally, recall that for left principal bundles, the structure equations state that the curvature $\Omega^{l}$ is given by

$$
\Omega^{l}=\mathrm{d} \Gamma^{l}-\frac{1}{2}\left[\Gamma^{l}, \Gamma^{l}\right]^{\hat{n}} .
$$

To compute the curvature, it is convenient to rewrite $\Gamma^{l}$ given by (12.1) intrinsically as

$$
\Gamma^{l}=\operatorname{pr}_{1}^{*} \rho-\left(\operatorname{pr}_{1}^{*} \text { Ad. }\right)\left(\operatorname{pr}_{2}^{*} \alpha\right)
$$

where the dot indicates a blank variable. Then we get

$$
\begin{aligned}
& \Omega^{l}=\mathbf{d} \Gamma^{l}-\frac{1}{2}\left[\Gamma^{l}, \Gamma^{l}\right]^{\wedge} \\
& =\mathbf{d p r}_{1}^{*} \rho-\frac{1}{2}\left[\operatorname{pr}_{1}^{*} \rho, \operatorname{pr}_{1}^{*} \rho\right]^{\wedge}-\mathbf{d}\left(\operatorname{pr}_{1}^{*} \mathrm{Ad} \cdot\left(\operatorname{pr}_{2}^{*} \alpha\right)\right) \\
& +\left[\operatorname{pr}_{1}^{*} \rho, \operatorname{pr}_{1}^{*} \text { Ad. }\left(\operatorname{pr}_{2}^{*} \alpha\right)\right]^{-}-\frac{1}{2}\left[\operatorname{pr}_{1}^{*} \operatorname{Ad} \cdot\left(\operatorname{pr}_{2}^{*} \alpha\right), \operatorname{pr}_{1}^{*} \operatorname{Ad} \cdot\left(\operatorname{pr}_{2}^{*} \alpha\right)\right] \text {. }
\end{aligned}
$$

The first two terms equal $\operatorname{pr}_{1}^{*}\left(\mathbf{d} \rho-\frac{1}{2}[\rho, \rho]\right)=0$ by (12.4). The third term equals

$$
-\mathbf{d}\left(\operatorname{pr}_{1}^{*} \text { Ad. }\left(\operatorname{pr}_{2}^{*} \alpha\right)\right)=-\left(\operatorname{pr}_{1}^{*} \mathbf{d} \text { Ad. }\right) \wedge \operatorname{pr}_{2}^{*} \alpha-\left(\operatorname{pr}_{1}^{*} \text { Ad. }\right)\left(\operatorname{pr}_{2}^{*} \mathbf{d} \alpha\right)
$$

However, if $\xi \in \mathfrak{g}$, we have

$$
(\mathbf{d A d} \text {. })(g) \cdot T_{e} R_{g} \xi=\left.\frac{d}{d t}\right|_{t=0} \operatorname{Ad}_{(\exp t \xi) g}=\operatorname{ad}_{\xi} \circ \operatorname{Ad}_{g}
$$


and therefore if $\left(u_{g}^{1}, v_{m}^{1}\right),\left(u_{g}^{2}, v_{m}^{2}\right) \in T_{g} G \times T_{m} M$ we get by (12.9)

$$
\begin{aligned}
-\mathbf{d}\left(\operatorname{pr}_{1}^{*} \operatorname{Ad} .\left(\operatorname{pr}_{2}^{*} \alpha\right)\right)(g, m)\left(\left(u_{g}^{1}, v_{m}^{1}\right),\left(u_{g}^{2}, v_{m}^{2}\right)\right) \\
=\quad-\left(\operatorname{ad}_{\rho\left(u_{g}^{1}\right)} \circ \operatorname{Ad}_{g}\right)\left(\alpha(m) \cdot v_{m}^{2}\right)+\left(\operatorname{ad}_{\rho\left(u_{g}^{2}\right)} \circ \operatorname{Ad}_{g}\right)\left(\alpha(m) \cdot v_{m}^{1}\right) \\
\quad-\operatorname{Ad}_{g}\left(\mathbf{d} \alpha(m)\left(v_{m}^{1}, v_{m}^{2}\right)\right) \\
=\quad-\left[\rho\left(u_{g}^{1}\right), \operatorname{Ad}_{g}\left(\alpha(m) \cdot v_{m}^{2}\right)\right]+\left[\rho\left(u_{g}^{2}\right), \operatorname{Ad}_{g}\left(\alpha(m) \cdot v_{m}^{1}\right)\right] \\
\quad-\operatorname{Ad}_{g}\left(\mathbf{d} \alpha(m)\left(v_{m}^{1}, v_{m}^{2}\right)\right) \\
=\quad-\left[\operatorname{pr}_{1}^{*} \rho,\left(\operatorname{pr}_{1}^{*} \operatorname{Ad}^{2}\right)\left(\operatorname{pr}_{2}^{*} \alpha\right)\right](g, m)\left(\left(u_{g}^{1}, v_{m}^{1}\right),\left(u_{g}^{2}, v_{m}^{2}\right)\right) \\
\quad-\left(\left(\operatorname{pr}_{1}^{*} \operatorname{Ad} .\right)\left(\operatorname{pr}_{2}^{*} \mathbf{d} \alpha\right)\right)(g, m)\left(\left(u_{g}^{1}, v_{m}^{1}\right),\left(u_{g}^{2}, v_{m}^{2}\right)\right) .
\end{aligned}
$$

Therefore, the first summand in (12.11) of the third term in (12.8) cancels the fourth term in (12.8) and we get

$$
\Omega^{l}=-\left(\operatorname{pr}_{1}^{*} \text { Ad. }\right)\left(\operatorname{pr}_{2}^{*}\left(\mathbf{d} \alpha+\frac{1}{2}[\alpha, \alpha]\right)\right) .
$$

Proposition 12.1 The curvature of the connection one-form $\Gamma^{l} \in \Omega^{1}(G \times M ; \mathfrak{g})$ given by (12.1) has the expression (12.12). by

If we assume that $M \times G \rightarrow M$ is a right action then $\Gamma^{r} \in \Omega^{1}(M \times G$, $\mathfrak{g})$ given

$$
\Gamma^{r}(m, g)\left(v_{m}, u_{g}\right)=T_{g} L_{g^{-1}}\left(u_{g}\right)-\operatorname{Ad}_{g^{-1}}\left(\alpha(m) \cdot v_{m}\right)
$$

is a right connection one-form whose curvature is given by

$$
\Omega^{r}=-\left(\operatorname{pr}_{1}^{*} \text { Ad. } \circ \operatorname{Inv}\right)\left(\operatorname{pr}_{2}^{*}\left(\mathbf{d} \alpha-\frac{1}{2}[\alpha, \alpha]\right)\right) .
$$

Here, Inv denotes the inversion map. The relative sign change occurs, since for right bundles and right connections, the structure equations are $\mathbf{d} \Gamma^{r}+(1 / 2)\left[\Gamma^{r}, \Gamma^{r}\right]^{\hat{}}=$ $\Omega^{r}$.

Corollary 12.2 The connection $\Gamma^{l}$ (respectively $\Gamma^{r}$ ) is flat if and only if $\mathbf{d} \alpha+$ $(1 / 2)[\alpha, \alpha]=0$ (respectively $\left.\mathbf{d} \alpha-(1 / 2)[\alpha, \alpha]^{\prime}=0\right)$.

Now recall that a principal connection is flat if and only if its horizontal subbundle is integrable. If $f: M \rightarrow G$, we will denote, following Kolár, Michor, and Slovák [1993], by $\delta^{l} f, \delta^{r} f \in \Omega^{1}(M ; \mathfrak{g})$ the left and right logarithmic derivatives of $f$ :

$$
\delta^{l} f(m)=T_{f(m)} L_{f(m)^{-1}} \circ T_{m} f=f^{*} \lambda, \quad \delta^{r} f(m)=T_{f(m)} R_{f(m)^{-1}} \circ T_{m} f=f^{*} \rho .
$$

Note that $\delta^{l} f(m)=\operatorname{Ad}_{f(m)^{-1}} \delta^{r} f(m)$. The following formulae are direct consequences of the definitions. If $f, h: M \rightarrow G$ we have

$$
\begin{array}{r}
\delta^{l}(f h)(m)=\operatorname{Ad}_{h(m)^{-1}} \delta^{l} f(m)+\delta^{l} h(m), \\
\delta^{r}(f h)(m)=\delta^{r} f(m)+\operatorname{Ad}_{f(m)} \delta^{r} h(m) .
\end{array}
$$


Denoting by $f^{-1}$ the map sending $m$ to $f(m)^{-1}$ we get

$$
\begin{gathered}
\delta^{l} f^{-1}(m)=-\delta^{r} f(m)=-\operatorname{Ad}_{f(m)} \delta^{l} f(m), \\
\delta^{r} f^{-1}(m)=-\delta^{l} f(m)=-\operatorname{Ad}_{f(m)^{-1}} \delta^{r} f(m) .
\end{gathered}
$$

These formulae combine to give:

$$
\begin{array}{r}
\delta^{l}\left(f h^{-1}\right)(m)=\operatorname{Ad}_{h(m)}\left(\delta^{l} f(m)-\delta^{l} h(m)\right), \\
\delta^{r}\left(f h^{-1}\right)(m)=\delta^{r} f(m)-\operatorname{Ad}_{f(m)} \operatorname{Ad}_{h(m)^{-1}} \delta^{r} h(m), \\
\delta^{l}\left(f^{-1} h\right)(m)=-\operatorname{Ad}_{h(m)^{-1}} \operatorname{Ad}_{f(m)} \delta^{l} f(m)+\delta^{l} h(m), \\
\delta^{r}\left(f^{-1} h\right)(m)=-\operatorname{Ad}_{f(m)^{-1}}\left(\delta^{r} h(m)-\delta^{r} f(m)\right) .
\end{array}
$$

The following Corollary may also be found in Sternberg [1963].

Corollary 12.3 For any smooth map $f: M \rightarrow G$, its logarithmic derivatives satisfy

$$
\begin{aligned}
& \mathbf{d} \delta^{l} f+\frac{1}{2}\left[\delta^{l} f, \delta^{l} f \hat{]}=0,\right. \\
& \mathbf{d} \delta^{r} f-\frac{1}{2}\left[\delta^{r} f, \delta^{r} f\right]=0 .
\end{aligned}
$$

Conversely, given a one-form $\alpha \in \Omega^{1}(M ; \mathfrak{g})$ satisfying $\mathbf{d} \alpha+(1 / 2)[\alpha, \alpha]=0$ (respectively $\left.\mathbf{d} \alpha-(1 / 2)[\alpha, \alpha]^{\prime}=0\right)$ for every $m \in M$ there is an open set $U \subset M, m \in U$ and a smooth function $f: U \rightarrow G$ such that $\delta^{l} f=\alpha \mid U$ (respectively $\delta^{r} f=\alpha \mid U$ ). If $M$ is simply connected we can take $U=M$. In this case, the map $f$ is uniquely determined up to multiplication on the left by a fixed group element.

Proof Given $f: M \rightarrow G$ consider the left principal connection $\Gamma^{l}$ defined by $\alpha=\delta^{l} f$ on the trivial bundle $\mathrm{pr}_{2}: G \times M \rightarrow M$. By (12.2), its horizontal subbundle $H^{l}$ equals

$$
H_{(g, m)}^{l}=\left\{\left(T_{m}\left(L_{g f(m)^{-1}} \circ f\right)\left(v_{m}\right), v_{m}\right) \mid v_{m} \in T_{m} M\right\} .
$$

This is, however, obviously integrable, the leaf through $(g, m)$ being

$$
\mathcal{H}_{(g, m)}^{l}=\left\{\left(g f(m)^{-1} f(x), x\right) \mid x \in M\right\} .
$$

Therefore, the curvature $\Omega^{l}$ vanishes and (12.24) holds by Corollary 4.2. Note that $\mathcal{H}_{(f(m), m)}^{l}=\operatorname{graph} f$.

Conversely, assume $\alpha \in \Omega^{1}(M ; \mathfrak{g})$ satisfies $\mathbf{d} \alpha+(1 / 2)[\alpha, \alpha]=0$. By Corollary 4.2 the connection $\Gamma^{l}$ it defines is flat and therefore its horizontal subbundle $H$, given by $(12.2)$, is integrable. Let $\mathcal{H}$ be one of the leaves of the induced foliation. Then $\mathrm{pr}_{2}: \mathcal{H} \rightarrow M$ is a smooth covering space, so in particular, if $m \in M$ there are open sets $U \subset M, m \in U$, and $V \subset \mathcal{H}$ such that $\mathrm{pr}_{2}: V \rightarrow U$ is a diffeomorphism. Let $x \in U \mapsto(f(x), x) \in V$ be its inverse, which thus defines a smooth map $f: U \rightarrow G$. We claim that $\delta^{l} f=\alpha \mid U$. Indeed, $\left(T_{x} f\left(v_{x}\right), v_{x}\right) \in T_{(f(x), x)}, \mathcal{H}=H_{(f(x), x)}^{l}$ so by $(12.2),\left(T_{x} f\left(v_{x}\right), v_{x}\right)=\left(T_{e} L_{f(x)}\left(\alpha(x) \cdot v_{x}\right), v_{x}\right)$, whence $\alpha(x)=T_{f(x)} L_{f(x)^{-1}} \circ T_{x} f=$ $\delta^{l} f$. 
If $M$ is simply connected the covering $\operatorname{pr}_{2}: \mathcal{H} \rightarrow M$ is necessarily a homeomorphism and hence a diffeomorphism. The open set $U$ can therefore be chosen to equal $M$. Now assume that there are two functions $f, h: M \rightarrow G$ such that $\alpha=\delta^{l} f(m)=\delta^{l} h(m)$. By (12.20), we conclude that $\delta^{l}\left(f h^{-1}\right)(m)=0$, i.e. that $T_{m}\left(f h^{-1}\right)=0$ for all $m \in M$. By connectedness of $M$, this implies that $f h^{-1}: M \rightarrow G$ is a constant function, i.e. there is some $g \in G$ such that $f(m) h(m)^{-1}=g$ for all $m \in M$, which is equivalent to $f=L_{g} \circ h$.

Proof of Proposition 5.1 Take in Corollary 4.3, $M=U, f=g$, and evaluate (12.24) on the basis vector fields $(\partial / \partial t, \partial / \partial \varepsilon)$. Since $[\partial / \partial t, \partial / \partial \varepsilon]=0$, we get

$$
\begin{array}{r}
\frac{\partial}{\partial t}\left[\delta^{l} g\left(\frac{\partial}{\partial \varepsilon}\right)\right]-\frac{\partial}{\partial \varepsilon}\left[\delta^{l} g\left(\frac{\partial}{\partial t}\right)\right]+\frac{1}{2}\left[\delta^{l} g\left(\frac{\partial}{\partial t}\right), \delta^{l} g\left(\frac{\partial}{\partial \varepsilon}\right)\right] \\
-\frac{1}{2}\left[\delta^{l} g\left(\frac{\partial}{\partial \varepsilon}\right), \delta^{l} g\left(\frac{\partial}{\partial t}\right)\right]=0 .
\end{array}
$$

However, by (12.15)

$$
\delta^{l} g\left(\frac{\partial}{\partial t}\right)=T L_{g(t, \varepsilon)^{-1}} \frac{\partial g(t, \varepsilon)}{\partial t}=\xi(t, \varepsilon),
$$

and similarly $\delta^{l} g(\partial / \partial \varepsilon)=\eta(t, \varepsilon)$, so that (12.28) becomes

$$
\frac{\partial \eta}{\partial t}-\frac{\partial \xi}{\partial \varepsilon}+\frac{1}{2}[\xi(t, \varepsilon), \eta(t, \varepsilon)]-\frac{1}{2}[\eta(t, \varepsilon), \xi(t, \varepsilon)]=0,
$$

which is equivalent to (5.1).

Conversely, given $U \subset \mathbb{R}^{2}$ simply connected and $\xi, \eta: U \rightarrow \mathfrak{g}$ satisfying (5.1), define $\alpha \in \Omega^{1}(U ; \mathfrak{g})$ by $\alpha=\xi(t, \varepsilon) d t+\eta(t, \varepsilon) d \varepsilon$. Then by $(5.1)$

$$
\mathbf{d} \alpha+\frac{1}{2}[\alpha, \alpha]=\left(-\frac{\partial \xi}{\partial \varepsilon}+\frac{\partial \eta}{\partial t}\right) d t \wedge d \varepsilon+\left(\frac{1}{2}[\xi, \eta]-\frac{1}{2}[\eta, \xi]\right) d t \wedge d \varepsilon=0 .
$$

By Corollary 4.3 there is a function $g: U \rightarrow G$ such that $\delta^{l} g=\alpha$ which, in view of the computations above, is equivalent to $\xi(t, \varepsilon)=T L_{g(t, \varepsilon)^{-1}}(\partial g(t, \varepsilon) / \partial t)$ and $\eta(t, \varepsilon)=T L_{g(t, \varepsilon)^{-1}}(\partial g(t, \varepsilon) / \partial \varepsilon)$.

We remark that formula (5.1) can also be deduced from the expression of the complete left trivialization of elements of TTG using the ideas in Marsden, Ratiu, and Raugel [1991]. If $V \in T T G$ is represented as an element of $G \times \mathfrak{g} \times \mathfrak{g} \times \mathfrak{g}$, its expression equals $\left(g, \eta, T_{g} L_{g^{-1}} \dot{g}(0), \dot{\eta}(0)+\left[T_{g} L_{g^{-1}} \dot{g}(0), \eta(0)\right]\right)$, where $V$ is represented as $V=\left.\left.(d / d s)\right|_{s=0}(d / d t)\right|_{t=0} g(t) \exp s \eta(t)$ for curves $g(t)$ in $G, g(0)=g$, and $\eta(t)$ in $\mathfrak{g}$. Formula (5.1) is then the fourth component of $V$ in this trivialization.

Acknowledgements We thank Miroslav Grmela, Darryl Holm, Alan Kaufman, Naomi Leonard, Peter Michor, Gloria Sanchez and the referees for helpful suggestions. We also thank the Fields Institute for providing the opportunity to meet in pleasant surroundings during which time some of the ideas in the paper were first worked out. We also thank the Erwin Schrödinger Institute for Mathematical Physics for their hospitality. 


\section{References}

Abarbanel, H.D.I., D.D. Holm, J.E. Marsden and T.S. Ratiu [1986] Nonlinear stability analysis of stratified fluid equilibria. Phil. Trans. R. Soc. Lond. A 318, 349-409; also Phys. Rev. Lett. 52 [1984] 2352-2355.

Abraham, R. and J.E. Marsden [1978] Foundations of Mechanics. Second Edition, Addison-Wesley Publishing Co., Reading, Mass..

Alekseevski, D.V. and P.W. Michor [1993] Characteristic classes and Cartan connections. preprint.

Arnold, V. [1988] Dynamical Systems III. Encyclopedia of Mathematics, SpringerVerlag.

Bloch, A.M., R.W. Brockett and T.S. Ratiu [1992] Completely integrable gradient flows. Comm. Math. Phys. 147, 57-74.

Bloch, A.M. and P.E. Crouch [1994] Reduction of Euler Lagrange problems for constrained variational problems and relation with optimal control problems. Proc. CDC, IEEE 33, 2584-2590.

Bloch, A.M., H. Flaschka, and T.S. Ratiu [1990] A convexity theorem for isospectral sets of Jacobi matrices in a compact Lie algebra. Duke Math. J. 61, 41-66.

Bloch, A.M., P.S. Krishnaprasad, J.E. Marsden and T.S. Ratiu [1991] Asymptotic stability, instability, and stabilization of relative equilibria. Proc. of $A C C$., Boston IEEE, 1120-1125.

Bloch, A.M., P.S. Krishnaprasad, J.E. Marsden and T.S. Ratiu [1994] Dissipation Induced Instabilities, Ann. Inst. H. Poincaré, Analyse Nonlineare 11, 37-90.

Bloch, A.M., P.S. Krishnaprasad, J.E. Marsden and G. Sánchez de Alvarez [1992] Stabilization of rigid body dynamics by internal and external torques. Automatica 28, 745-756.

Brockett, R.W. [1973] Lie theory and control systems defined on spheres. SIAM J. Appl. Math./ 23, 213-225.

Brockett, R.W. [1988] Dynamical systems that sort lists and solve linear programming problems. Proc. IEEE 27, 799-803 and Linear Algebra and its Appl. 146, (1991), 79-91.

Brockett, R.W. [1993] Differential geometry and the design of gradient algorithms. Proc. Symp. Pure Math., AMS 54, Part I, 69-92.

Brockett, R.W. [1994] The double bracket equation as a solution of a variational problem. Fields Institute Comm., 3, 69-76.

Chandrasekhar, K. [1977] Ellipsoidal Figures of Equilibrium. Dover. 
Chern, S.J. and J.E. Marsden [1990] A note on symmetry and stability for fluid flows. Geo. Astro. Fluid. Dyn. 51, 1-4.

Chetaev, N.G. [1961] The stability of Motion. Trans. by M. Nadler, Pergamon Press, New York.

Crouch, P.E. [1981] Geometric structures in systems theory. IEEE Proc, Part D, No 5128.

Ebin, D.G. and J.E. Marsden [1970] Groups of diffeomorphisms and the motion of an incompressible fluid. Ann. Math. 92, 102-163.

Giles, R., G. Patterson, A. Bagneres, R. Kotiuga, F. Humphrey and M. Mansuripur [1991] Micromagnetic simulations on the connection machine. Very Large Scale Computation in the 21st Century, J.P. Mesirov, ed., SIAM, 33-40.

Grmela, M. [1993a] Weakly nonlocal hydrodynamics. Phys. Rev. E 47, 351-365.

Grmela, M. [1984] Bracket formulation of dissipative fluid mechanics equations. Phys. Lett. A 102, 355-358.

Grmela, M. [1993b] Thermodynamics of driven systems. Phys. Rev. E 48, 919930.

Guckenheimer, J. and A. Mahalov [1992], Instability induced by symmetry reduction. Phys. Rev. Lett., 68, 2257-2260.

Hahn, W. [1967] Stability of Motion. Springer-Verlag, New York.

Haller, G. [1992] Gyroscopic stability and its loss in systems with two essential coordinates. Int. J. Nonlinear Mech. 27, 113-127.

Helman, J.S., H.B. Braun, J.S. Broz, and W. Baltensperger [1991] General solution to the Landau-Lifschitz-Gilbert equations linearized around a Bloch wall. Phys. Rev. B 43, 5908-5914.

Holm, D.D., J.E. Marsden, T.S. Ratiu and A. Weinstein [1985] Nonlinear stability of fluid and plasma equilibria. Phys. Rep. 123, 1-116.

Kammer, D. C., and G.L. Gray [1993] A nonlinear control design for energy sink simulation in the Euler-Poinsot problem. J. Astron. Sci. 41, 53-72.

Kandrup, H.E. [1991] The secular instability of axisymmetric collisionless star cluster. Astrophy. J. 380, 511-514.

Kandrup, H.E. and P. Morrison [1992] Hamiltonian structure of the Vlasov-Einstein system and the problem of stability for spherical relativistic star clusters. preprint..

Kaufman, A.N. [1984] Dissipative Hamiltonian systems: A unifying principle. Physics Letters A 100, 419-422. 
Kaufman, A.N. [1985] Lorentz-covariant dissipative Lagrangian systems. Physics Letters A 109, 87-89.

Kolár, I., P.W. Michor, J. Slovák [1993] Natural Operations in Differential Geometry. Springer-Verlag.

Knobloch, E., A. Mahalov, and J.E. Marsden [1994] Normal Forms for threedimensional Parametric Instabilities in Ideal Hydrodynamics, Physica D 73, 49-81.

Krein, M.G. [1950] A generalization of some investigations of linear differential equations with periodic coefficients. Doklady Akad. Nauk SSSR N.S. 73, 445-448.

Krishnaprasad, P.S. [1985] Lie-Poisson structures, dual-spin spacecraft and asymptotic stability. Nonl. An. Th. Meth. and Appl. 9, 1011-1035.

LaSalle, J.P. and S. Lefschetz [1963] Stability by Lyapunov's direct method. Academic Press, New York.

Lewis, D.K. [1992] Lagrangian block diagonalization. Dyn. Diff. Eqn's. 4 1-42.

Lewis, D., T.S. Ratiu, J.C. Simo and J.E. Marsden [1992] The heavy top, a geometric treatment. Nonlinearity 5, 1-48.

Lewis, D.K. and J.C. Simo [1990] Nonlinear stability of rotating pseudo-rigid bodies. Proc. Roy. Soc. Lon. A 427, 281-319.

MacKay, R. [1991] Movement of eigenvalues of Hamiltonian equilibria under nonHamiltonian perturbation. Phys. Lett. A155, 266-268.

Marsden, J.E. [1992], Lectures on Mechanics London Mathematical Society Lecture note series, 174, Cambridge University Press.

Marsden, J.E. and T.S. Ratiu [1992] Symmetry and Mechanics. Texts in Applied Mathematics, 17, Springer-Verlag.

Marsden, J.E., T.S. Ratiu and G. Raugel [1991] Symplectic connections and the linearization of Hamiltonian systems. Proc. Roy. Soc. Ed. A 117, 329-380

Marsden, J.E., T.S. Ratiu and A. Weinstein [1984] Semi-direct products and reduction in mechanics, Trans. Am. Math. Soc. 281, 147-177.

Marsden, J.E. and J. Scheurle [1993a] Lagrangian reduction and the double spherical pendulum. ZAMP 44, 17-43.

Marsden, J.E. and J. Scheurle [1993b] The reduced Euler-Lagrange equations. Fields Institute Comm. 1, 139-164.

Marsden, J.E. and A. Weinstein [1982] The Hamiltonian structure of the MaxwellVlasov equations. Physica D 4, 394-406. 
Morrison, P.J. [1980] The Maxwell-Vlasov equations as a continuous Hamiltonian system. Phys. Lett. A 80, 383-386.

Morrison, P.J. [1982] Poisson Brackets for fluids and plasmas, in Mathematical Methods in Hydrodynamics and Integrability in Related Dynamical Systems. AIP Conf. Proc. 88, M. Tabor and Y.M. Treve (eds.), La Jolla, Calif.

Morrison, P.J. [1986] A paradigm for joined Hamiltonian and dissipative systems. Physica D 18, 410-419.

Morrison, P.J. and M. Kotschenreuther [1989] The free energy principle, negative energy modes and stability, Proc 4th Int. Workshop on Nonlinear and Turbulent Processes in Physics, World Scientific Press.

Newcomb, W.A. [1962] Lagrangian and Hamiltonian methods in Magnetohydrodynamics. Nuc. Fusion Suppl., part 2, 451-463.

O’Dell, T.H. [1981] Ferromagnetodynamics. John Wiley and Sons.

O'Reilly, O, N.K. Malhotra and N.S. Namamchchivaya [1993] Destabilization of the equilibria of reversible dynamical systems, preprint.

Pego, R.L. and M.I. Weinstein [1992] Eigenvalues and instabilities of solitary waves. Phil. Trans. Roy. Soc. Lon. 340, 47-94.

Poincaré, H. [1885] Sur l'équilibre d'une masse fluide animée d'un mouvement de rotation. Acta. Math. 7, 259.

Poincaré, H. [1892] Les formes d'équilibre d'une masse fluide en rotation. Revue Générale des Sciences 3, 809-815.

Poincaré, H. [1901] Sur la stabilité de l'équilibre des figures piriformes affectées par une masse fluide en rotation. Philosophical Transactions A 198, 333-373.

Poincaré, H. [1910] Sur la precession des corps deformables. Bull Astron 27, 321356.

Posbergh, T.A. [1994] The damped vibration absorber as a feedback control problem. Preprint.

Posbergh, T.A. and R. Zhao [1993] Stabilization of the uniform rotation of a rigid body by the energy-momentum method. Fields Inst. Comm. 1, 263-280.

Riemann, B. [1860] Untersuchungen über die Bewegung eines flüssigen gleichartigen Ellipsoides. Abh. d. Königl. Gesell. der Wiss. zu Göttingen 9, $3-36$.

Routh, E.J. [1877] Stability of a given state of motion. Reprinted in Stability of Motion, ed. A.T. Fuller, Halsted Press, New York, 1975. 
Seliger, R.L. and G.B. Whitham [1968] Variational principles in continuum mechanics, Proc. Roy. Soc. Lond. 305, 1-25.

Shepherd, T.G. [1992] Extremal properties and Hamiltonian structure of the Euler equations. Topological Aspects of the Dynamics of Fluids and Plasmas, H.K. Moffatt et. al., eds, KIuwer, 275-292.

Simo, J.C., D.K. Lewis and J.E. Marsden [1991] Stability of relative equilibria I: The reduced energy momentum method. Arch. Rat. Mech. Anal. 115, 15-59.

Simo, J.C., T.A. Posbergh and J.E. Marsden [1990] Stability of coupled rigid body and geometrically exact rods: block diagonalization and the energymomentum method. Physics Reports 193, 280-360.

Simo, J.C., T.A. Posbergh and J.E. Marsden [1991] Stability of relative equilibria II: Three dimensional elasticity. Arch. Rat. Mech. Anal. 115, 61-100.

Sri Namachchivaya, N., S.T. Ariaratnam [1985] On the dynamic stability of gyroscopic systems. SM Archives 10, 313-355.

Sternberg, S. [1963] Lectures on Differential Geometry. Prentice-Hall. (Reprinted by Chelsea, 1983)

Taussky, O. [1961] A Generalization of a Theorem of Lyapunov. SIAM J. Appl. Math 9, 640-643.

Thomson, L. and P.G. Tait [1879] Treatise on Natural Philosophy. Cambridge Univ. Press.

Touma, J. and J. Wisdom [1994] Lie-Poisson integrators for rigid body dynamics in the solar system. Astro. J. 107, 1189.

Touma, J. and J. Wisdom [1994] Evolution of the Earth-Moon system. Astro. J. 108, 1943.

Turski, L.A. and A.N. Kaufman [1987] Canonical-dissipative formulation of relativistic plasma kinetic theory with self-consistent Maxwell field. Physics Lett. A 120, 331-333.

Vallis, G.K., G.F. Carnevale, and W.R. Young [1989] Extremal energy properties and construction of stable solutions of the Euler equations. J. Fluid Mech. 207, 133-152.

van Gils, S.A., M. Krupa and W.F. Langford [1990] Hopf bifurcation with nonsemisimple 1:1 resonance. Nonlinearity 3, 825-830.

Vershik, A.M. and L.D. Faddeev [1981] Lagrangian mechanics in invariant form. Sel. Math. Sov. 1, 339-350.

Wang, L.S. and P.S. Krishnaprasad [1992] Gyroscopic control and stabilization. J. Nonlinear Sci. 2, 367-415. 
Ziegler, H. [1956] On the concept of elastic stability. Adv. Appl. Mech. 4, 351-403.

Communicated by S.-T. Yau 